\title{
Generation of mechanical force by grafted polyelectrolytes in an electric field
}

\author{
N. V. Brilliantov \\ Department of Mathematics, University of Leicester, Leicester LE1 7RH, United Kingdom \\ Yu. A. Budkov \\ G.A. Krestov Institute of Solution Chemistry, Russian Academy of Sciences, Akademicheskaya Street 1, 153045 Ivanovo, Russia \\ and Department of Applied Mathematics, National Research University Higher School of Economics, Moscow, Russia \\ C. Seidel \\ Max Planck Institute of Colloids and Interfaces, Science Park Golm, D-14424 Potsdam, Germany
}

(Received 12 October 2015; published 16 March 2016)

\begin{abstract}
We study theoretically and by means of molecular dynamics (MD) simulations the generation of mechanical force by grafted polyelectrolytes in an external electric field, which favors its adsorption on the grafting plane. The force arises in deformable bodies linked to the free end of the chain. Varying the field, one controls the length of the nonadsorbed part of the chain and hence the deformation of the target body, i.e., the arising force too. We consider target bodies with a linear force-deformation relation and with a Hertzian one. While the first relation models a coiled Gaussian chain, the second one describes the force response of a squeezed colloidal particle. The theoretical dependences of generated force and compression of the target body on an applied field agree very well with the results of MD simulations. The analyzed phenomenon may play an important role in future nanomachinery, e.g., it may be used to design nanovices to fix nanosized objects.
\end{abstract}

DOI: 10.1103/PhysRevE.93.032505

\section{INTRODUCTION}

Due to its obvious importance for applications, the response of polyelectrolytes to external electric fields has been of high scientific interest for the past few decades (see, e.g., [1-18]). Moreover, novel experimental techniques that allow exploration of a single polymer chain have aided developments in this area [11]. In fact, the ability of polyelectrolyte chains to adapt their conformation in external electric fields, i.e., to change between expanded and contracted states when the applied field varies, is an important property. It may be used in future nanomachinery: Possible examples of such nanodevices may be nanovices or nanonippers manipulated by an electric field.

Suppose one end of a chain is fixed on a plane (i.e., the polyelectrolyte is grafted) while the other end is linked to a nanosized (target) body that can suffer deformation. If the polyelectrolyte is exposed to an external electric field that favors adsorption at the grafting plane, its conformation will be determined by both the field and the restoring force exerted by the deformed target body on the chain (see Fig. 1). Increased adsorption of the polyelectrolyte in response to a changing electric field will cause a deformation of the target body and give rise to a force acting between the chain and target. More precisely, the force will depend on both the magnitude of the deformation and the specific force-deformation relation of the target body. Hence, by applying an electric field, one can manipulate the conformation of polyelectrolyte chains as well as the force affecting the target body.

The nature of target bodies may be rather different however; the most important ones with respect to possible applications seem to be either polymer chains or nanoparticles, e.g., colloidal particles (see Figs. 1 and 2). In the latter case the force-deformation relation is given by the Hertzian law, which accurately describes the elastic response of squeezed nanoparticles $[19,20]$. On the other hand, polymer chains can exhibit coiled states with a linear force-deformation relation or stretched conformations with a nonlinear relation (see, e.g., [21]). To describe the phenomenon it is necessary to express the size of the polyelectrolyte chain as well as the force acting on the target body as a function of the applied electric field.

In the present study we address the problem theoretically and numerically by means of molecular dynamics (MD) simulations. We analyze a model of a polyelectrolyte chain grafted to a plane, linked by its free end to a deformable target body and exposed to an external electric field. The target body is modeled by linear or nonlinear springs with corresponding force-deformation relations. A time-independent electric field is applied perpendicular to the grafting plane so that it favors complete polyelectrolyte adsorption on the plane. For simplicity we consider a salt-free solution, i.e., there are only counterions that compensate for the charge of the chain. For intermediate and strong electric fields (the definition is given below), additional salt leads to a renormalization of the surface charge. This happens because the salt co-ions simply screen the plane, leaving the qualitative nature of the phenomenon unchanged. Hence the salt-free case addressed here is the basic one, which allows a simpler analytical treatment. The general case of a solution with additional salt ions will be studied elsewhere [22].

Counterions having the same charge sign as the grafting plane are repelled, leaving the chain unscreened see (see Fig. 1). This feature is dominating if the specific volume per chain is not small and the electric field is not weak. In weak fields a noticeable fraction of counterions is located close to the chain, which leads to a partial screening of the external field and of the Coulomb interactions between monomers. Here we consider systems with a large specific volume and with fields that are not very weak. The screening of the chain in this case 

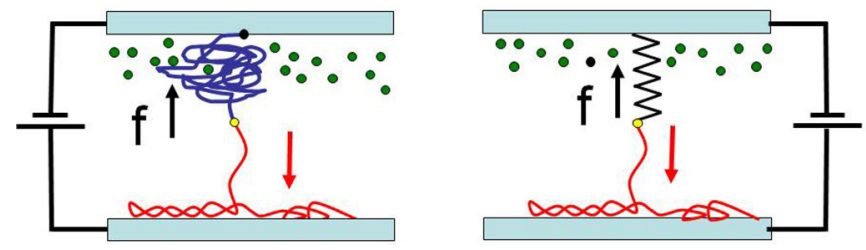

FIG. 1. Illustration of the generation of mechanical force by an electric field. The electric field causes chain contraction indicated by down arrows. The restoring force $f$ of the deformed target body (up arrows) can be both linear and nonlinear, depending on the nature of the target body. The right panel shows that the target body is modeled by a spring.

may be treated as a small perturbation. We study the static case when the current across the system is zero. It is noteworthy that for the specific volumes and magnitudes of the electric field addressed here, MD simulations demonstrate a lack of the counterion screening even at finite electric current [13].

Here we present a first-principle theory of the phenomena and compare theoretical predictions with MD simulations results. We observe quantitative agreement between theory and MD data for all magnitudes of electric field, except very weak fields when screening of the polyelectrolyte becomes significant. The simpler problem of the conformation of a grafted polyelectrolyte exposed to a constant force in an electric field has been explored theoretically and numerically in a previous study [12]. In Refs. [12,13] we also reported some simulation results for a chain linked to a deformable target body along with our previous simpler theory for the restoring force. In the present study we develop a first-principles theory, based on a unified approach that describes the adsorbed part of the chain as well as the bulk part under the action of the force from the target body.

The paper is organized as follows. In Sec. II we present our analytical theory, where we calculate the free energy of the chain and the force acting on the target body. In Sec. III the numerical setup is discussed and in Sec. IV we present the MD results and compare them with our theoretical predictions. We summarize our findings in Sec. V.

\section{THEORY}

We consider a system, composed of a chain of $N_{0}+1$ monomers, which is anchored to a planar surface at $z=0$.
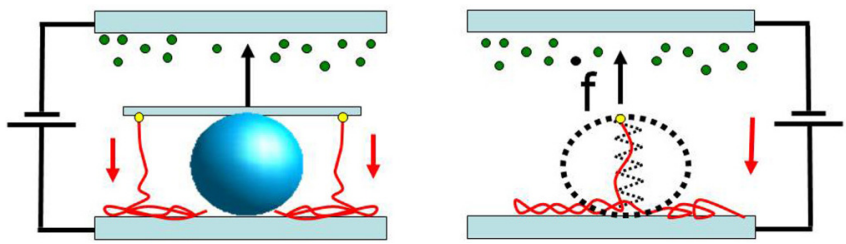

FIG. 2. Illustration of the work principle of the nanovice. The target particle, i.e., the colloidal particle, is fixed at sufficiently strong fields due to the polyelectrolyte chain compression; it will be released at zero field. The restoring force $f$ corresponds in this case to the Hertzian response of a compressed sphere. To illustrate a possible device, two polyelectrolyte chains are sketched, although only one chain, linked to the Hertzian spring, was used in the simulations reported (see the right panel).

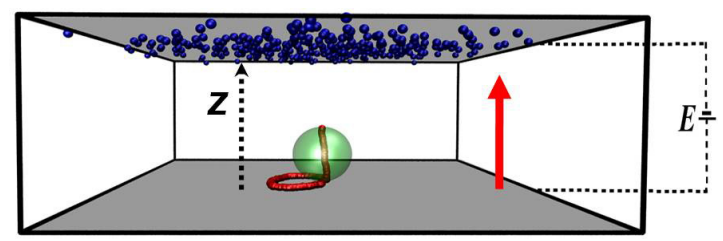

FIG. 3. Typical simulation snapshot of a grafted polyelectrolyte exposed to electrical field $\tilde{E}=1$, perpendicular to the grafting plane, and coupled to a deformable colloidal particle of diameter $h_{0}=80 \mathrm{~b}$. The action of the particle is modeled by a Hertzian spring with spring constant $\tilde{\kappa}=\kappa b^{5 / 2} / k_{B} T=1$. The total length of the chain is $N_{0}=320$. As can be seen from the figure, for the system parameters addressed, counterions are practically decoupled from the polyelectrolyte.

The anchoring end monomer is uncharged, while each of the remaining $N_{0}$ beads carries the charge $-q e(e>0$ is the elementary charge); $N_{0}$ counterions of charge $+q e$ make the system neutral. The external electric field $\mathbf{E}$ acts perpendicular to the plane and favors the adsorption of the chain (Fig. 3). The free end of the polyelectrolyte is linked to a deformable body, modeled by a spring with various force-deformation relations. We study a few different cases. The reaction force $f$ and the energy of deformation $U_{\text {sp }}$ for a linear spring reads

$$
f=-\kappa\left(h-h_{0}\right), \quad U_{\mathrm{sp}}=\frac{\kappa}{2}\left(h-h_{0}\right)^{2} .
$$

Here $\kappa$ is the elastic constant of the spring and $h$ and $h_{0}$ are the lengths of the deformed and undeformed springs, respectively. A linear force-deformation relation corresponds, for instance, to a target body given by a polymer chain in a coiled Gaussian state (see, e.g., [21]). The corresponding relation for a nonlinear spring has the form

$$
f=\kappa\left|h-h_{0}\right|^{\gamma} \operatorname{sgn}\left(h_{0}-h\right), \quad U_{\mathrm{sp}}=\frac{\kappa}{\gamma+1}\left|h-h_{0}\right|^{\gamma+1},
$$

where $\gamma>1$ characterizes the stiffness of the body, which may be, e.g., a polymer chain in a semistretched conformational state, i.e., in a state intermediate between a coiled and a stretched one. It is known that stretched polymer chains demonstrate much larger stiffness than Gaussian ones [21]. Hence, by varying the exponent $\gamma$ one can mimic different states of a chain. From the point of view of applications, it is worthwhile studying the special case of a Hertzian spring with $\gamma=3 / 2$, which corresponds to the elastic response of a squeezed nanoparticle [19,20], e.g., a colloidal particle

$$
\begin{aligned}
f & =\kappa\left(h_{0}-h\right)^{3 / 2} \theta\left(h_{0}-h\right), \\
U_{\text {sp }} & =\frac{2}{5} \kappa\left(h_{0}-h\right)^{5 / 2} \theta\left(h_{0}-h\right) .
\end{aligned}
$$

Here $h_{0}=d_{c}$ is the diameter of an unloaded colloidal particle and $h$ that of the deformed one. The unit Heaviside step function $\theta(x)$ reflects the fact that the Hertzian elastic respond arises for compressive deformations only. Although we performed MD simulations only for the above models of the elastic response, the theoretical analysis is given for the general case

$$
U_{\mathrm{sp}}=U_{\mathrm{sp}}\left(h-h_{0}\right), \quad f=-\frac{\partial}{\partial h} U_{\mathrm{sp}}\left(h-h_{0}\right),
$$


where again $h_{0}$ and $h$ are the sizes of undeformed and deformed target bodies, respectively.

To find the polyelectrolyte conformation in an electric field and the force acting on the target body we evaluate the conditional free energy of the system and minimize it with respect to relevant variables. Let the number of (charged) monomers adsorbed at the (oppositely charged) plane be $N_{s}$ so that $N=N_{0}-N_{s}$ is the number of monomers in the bulk. Let $z_{\text {top }}$ be the distance of the free chain end, linked to the target body, from the charged plane and $\mathbf{R}$ the end-to-end distance of the adsorbed polymer part of $N_{s}$ monomers. In what follows we compute the conditional free energy $F\left(N, z_{\text {top }}, R\right)$, which may be written as

$$
F\left(N, z_{\mathrm{top}}, R\right) \approx F_{b}+F_{s}+F_{b s},
$$

where $F_{b}=F_{b}\left(N, z_{\text {top }}\right)$ is the free energy of the system associated with the bulk part of the chain and the target body, $F_{s}=F_{s}\left(N_{s}, R\right)$ is the free energy of the adsorbed part of the chain, and $F_{b s}=F_{b s}\left(N, z_{\text {top }}, R\right)$ accounts for the interactions between the bulk and adsorbed parts. Minimizing then $F\left(N, z_{\text {top }}, R\right)$ with respect to $N, z_{\text {top }}$, and $R$ one can find the conformation of the chain and the force acting on the target body (see the detailed discussion below).

In the present study we focus on the range of parameters where the polyelectrolyte chain is weekly screened. This allows us to treat the interaction of the chain with counterions as a small perturbation and estimate it separately; this significantly simplifies calculations. In what follows we compute separately different parts of the free energy.

\section{A. Free energy of the bulk part of the chain}

For simplicity, we use the freely jointed chain model with $b$ being the length of the inter-monomer links, that is, the size of the monomer beads. The MD simulations discussed below provide a justification for this model. The location of all monomers of the chain is determined by $N_{0}$ vectors $\mathbf{b}_{i}=$ $\mathbf{r}_{i}-\mathbf{r}_{i+1}$, which join the centers of $(i+1)$ st and $i$ th monomers $\left(i=1,2, \ldots, N_{0}\right)$. It is convenient to enumerate the monomers, starting from the free end linked to the target body. Then the beads with numbers $1,2, \ldots, N$ refer to the bulk part of the chain and with numbers $N+1, N+2, \ldots, N_{0}$ to the adsorbed part. The $\left(N_{0}+1\right)$ st neutral bead is anchored to the surface. Let the centers of the adsorbed beads lie at the plane $z=$ $0 ;^{1}$ for simplicity the anchored bead is located at the origin $\mathbf{r}_{N_{0}+1}=0$. Then the location of the $k$ th bead of the bulk part $(k=1,2, \ldots, N)$ may be written as

$$
\begin{aligned}
\mathbf{r}_{k} & =\mathbf{r}_{k}-\mathbf{r}_{k+1}+\mathbf{r}_{k+1}-\mathbf{r}_{k+2} \cdots+\mathbf{r}_{N_{0}}-\mathbf{r}_{N_{0}+1}+\mathbf{r}_{N_{0}+1} \\
& =\sum_{s=k}^{N_{0}} \mathbf{b}_{s}=\sum_{s=k}^{N} \mathbf{b}_{s}+\sum_{s=N+1}^{N_{0}} \mathbf{b}_{s}=\mathbf{r}_{N+1}+\sum_{s=k}^{N} \mathbf{b}_{s},
\end{aligned}
$$

\footnotetext{
${ }^{1}$ Here we ignore the off-surface loops of the adsorbed part of the chain. These may be taken into account [12], but they do not give an important contribution to the total free energy for the range of parameters addressed here.
}

where $\mathbf{r}_{N+1}$ is the radius vector of the $(N+1)$ st bead, which is a surface bead; it is linked to the $N$ th bead, located in the bulk.

The intercenter distance of the $i$ th and $j$ th beads reads

$$
\mathbf{r}_{i j}=\sum_{s=i}^{j} \mathbf{b}_{s}
$$

where each of the vectors $\mathbf{b}_{s}$ has the same length $b$. Its orientation may be characterized by the polar $\theta_{s}$ and azimuthal $\psi_{s}$ angles, where the axis $O Z$ is directed perpendicular to the grafting plane (Fig. 3). Hence, the distances between the reference plane $z=0$ and the $k$ th bead, as well as between the plane and the top bead, are

$$
z_{k}=b \sum_{s=k}^{N} \cos \theta_{s}, \quad z_{\text {top }}=z_{1}=b \sum_{s=1}^{N} \cos \theta_{s} .
$$

The location of the top bead $z_{\text {top }}$, linked to the target body, determines its deformation and the elastic energy due to the body deformation

$$
U_{\mathrm{sp}}\left(z_{\mathrm{top}}\right)=U_{\mathrm{sp}}\left(z_{\mathrm{top}}-z_{\mathrm{top}, 0}\right), \quad f=-\frac{\partial U_{\mathrm{sp}}}{\partial z_{\mathrm{top}}} .
$$

Here $z_{\text {top }, 0}$ is the location of the top bead of the chain when the target body is not deformed. Because the chain is assumed to be weakly screened, here we ignore screening effects, which we estimate later as a perturbation. Then the potential of the external field $\varphi_{\text {ext }}$ depends on $z$ simply as $\varphi_{\text {ext }}(z)=-E z$, so the electrostatic energy of $k$ th bead associated with this field reads $-q e \varphi_{\mathrm{ext}}\left(z_{k}\right)=$ bqe $E \sum_{s=k}^{N} \cos \theta_{s}$. Hence the interaction energy of the bulk part of the chain with the external field has the form

$$
\begin{aligned}
H_{\mathrm{ext}} & =\sum_{k=1}^{N}-q e \varphi_{\mathrm{ext}}\left(z_{k}\right)=\text { bqeE } \sum_{k=1}^{N} \sum_{s=k}^{N} \cos \theta_{s} \\
& =b q e E \sum_{s=1}^{N} s \cos \theta_{s} .
\end{aligned}
$$

Now we need to take into account the electrostatic interactions between chain monomers. Because of vanishing screening we have

$$
H_{\text {self }, b}=\frac{1}{2} \sum_{i=1}^{N} \sum_{j=1, j \neq i}^{N} V\left(\mathbf{r}_{i}-\mathbf{r}_{j}\right)=\frac{1}{2} \sum_{i=1}^{N} \sum_{j=1, j \neq i}^{N} \frac{q^{2} e^{2}}{\varepsilon r_{i j}},
$$

where $\varepsilon$ is the dielectric permittivity of the solution. Using the Fourier transform of the Coulomb potential $V(r)=$ $e^{2} q^{2} / \varepsilon r$ and the expression (6) for the intermonomer distances, Eq. (10) may be recast in the form (see the Appendix, Sec. 1)

$$
\begin{aligned}
H_{\text {self }, b}= & \frac{q^{2} e^{2}}{2 \varepsilon} \sum_{s_{1} \neq s_{2}} \int \frac{d \mathbf{k}}{(2 \pi)^{3}}\left(\frac{4 \pi}{k^{2}}\right) \\
& \times \exp \left(i \sum_{s=s_{1}}^{s_{2}} \mathbf{k}_{\perp} \cdot \mathbf{b}_{s}^{\perp}+k_{z} b_{s}^{z}\right),
\end{aligned}
$$


where $\mathbf{k}_{\perp}, \mathbf{b}_{s}^{\perp}$ and $k_{z}, b_{s}^{z}=b \cos \theta_{s}$ are, respectively, the transverse and longitudinal (parallel to the axis $O Z$ ) components of the vectors $\mathbf{k}$ and $\mathbf{b}_{s}$.

In the following we first compute the partition function associated with the bulk part of the chain. We impose the condition that the distance between the surface and the top bead, attached to the target body, is $z_{\text {top. }}$ Then the bulk part of the partition function reads

$$
\begin{aligned}
\mathcal{Z}_{b}\left(z_{\text {top }}\right)= & \int_{0}^{2 \pi} d \psi_{1} \cdots \int_{0}^{2 \pi} d \psi_{N} \int_{0}^{1} d \cos \theta_{1} \cdots \int_{0}^{1} d \cos \theta_{N} \\
& \times e^{-\beta U_{\mathrm{sp}}-\beta H_{\mathrm{self}, b}-\beta H_{\mathrm{ext}}} \delta\left(z_{\mathrm{top}}-b \sum_{s=1}^{N} \cos \theta_{s}\right) b
\end{aligned}
$$

where $\beta=1 / k_{B} T$, with $T$ being the temperature of the system and $k_{B}$ the Boltzmann constant; the energies $U_{\mathrm{sp}}, H_{\mathrm{ext}}$, and $H_{\text {self, } b}$ are defined by Eqs. (8)-(11) and the factor $b$ keeps $\mathcal{Z}_{b}$ dimensionless. In Eq. (12) we also assume that the vectors $\mathbf{b}_{s}$ cannot be directed downward $\left(\cos \theta_{s} \geqslant 0\right)$, which guarantees that the constraint $z_{s}>0, s=1, \ldots, N$, holds true; this has been confirmed in our MD simulations.

To proceed we assume that the value of $H_{\text {self }, b}$ may be approximated by its average over the angles $\psi_{1}, \ldots, \psi_{N}$, that is, $H_{\text {self }, b} \approx\left\langle H_{\text {self }, b}\right\rangle_{\psi}$, hence we assume that the transversal fluctuations of the polyelectrolyte chain are small. Then, with the use of (9), we rewrite Eq. (12) as

$$
\begin{aligned}
\mathcal{Z}_{b}\left(z_{\text {top }}\right)= & (2 \pi)^{N} \int_{0}^{1} d \eta_{1} \cdots \int_{0}^{1} d \eta_{N} \delta\left(\sum_{s=1}^{N} \eta_{s}-\tilde{z}_{\text {top }}\right) \\
& \times \exp \left[-\beta U_{\text {sp }}\left(z_{\text {top }}\right)-\tilde{E} \sum_{s=1}^{N} s \eta_{s}-\beta\left\langle H_{\text {self }, b}\right\rangle_{\psi}\right],
\end{aligned}
$$

where $\eta_{s}=\cos \theta_{s}, \tilde{z}_{\text {top }}=z_{\text {top }} / b, \tilde{E}=\beta q e E b$, and

$$
\begin{aligned}
\left\langle H_{\text {self }, b}\right\rangle_{\psi}= & \frac{q^{2} e^{2}}{2 \varepsilon} \sum_{s_{1} \neq s_{2}} \int \frac{d \mathbf{k}}{(2 \pi)^{3}}\left(\frac{4 \pi}{k^{2}}\right) \\
& \times\left\langle\exp \left(i \mathbf{k}_{\perp} \cdot \sum_{s=s_{1}}^{s_{2}} \mathbf{b}_{s}^{\perp}\right)\right\rangle_{\psi} \exp \left(i k_{z} b \sum_{s=s_{1}}^{s_{2}} \eta_{s}\right) .
\end{aligned}
$$

To evaluate the latter expression we exploit the approximation

$$
\eta_{s} \approx\left\langle\eta_{s}\right\rangle=\left\langle\cos \theta_{s}\right\rangle=\frac{z_{\text {top }}}{b N}
$$

which implies that $z_{\text {top }} \leqslant b N$ (recall that we consider a freely joined chain with constant links $b$ ) and that $\sum_{s=s_{1}}^{s_{2}} \eta_{s} \approx$ $z_{\text {top }}\left|s_{2}-s_{1}\right| / b N$. Referring for details to the Appendix, Sec. 1, we present here the result for $H_{\text {self }, b}$, averaged over transverse fluctuations:

$$
\beta\left\langle H_{\mathrm{self}, b}\right\rangle_{\psi}=\frac{l_{B} q^{2} N^{2}}{z_{\text {top }}}(\log N-1),
$$

where $l_{B}=e^{2} / \varepsilon k_{B} T$ is the Bjerrum length.

Using the integral representation of the $\delta$ function,

$$
\delta(x)=(2 \pi)^{-1} \int_{-\infty}^{+\infty} d \xi e^{i \xi x}
$$

we recast $\mathcal{Z}_{b}\left(z_{\text {top }}\right)$ in Eq. (13) in the form

$$
\mathcal{Z}_{b}\left(z_{\mathrm{top}}\right)=(2 \pi)^{N-1} e^{-\beta U_{\mathrm{sp}}-\beta\left\langle H_{\mathrm{self}, b}\right\rangle_{\psi}} \int_{-\infty}^{+\infty} d \xi e^{-i \xi \tilde{z}_{\mathrm{top}}+W(\xi)},
$$

where $W(\xi)$ contains the integration over $\eta_{1}, \ldots, \eta_{N}$. Its explicit expression is given in the Appendix, Sec. 2. For large $N \gg 1$, one can use the the steepest-descent method to estimate the above integral over $\xi$. Neglecting small terms, we finally obtain

$$
\mathcal{Z}_{b}\left(z_{\text {top }}\right) \approx(2 \pi)^{N-1} e^{-\beta U_{\mathrm{sp}}-\beta\left\langle H_{\text {self }, b}\right\rangle_{\psi}-\xi_{0} \tilde{z}_{\text {top }}+W\left(\xi_{0}\right)},
$$

where $\xi_{0}$ is the root of the saddle point equation $i z_{\text {top }}-$ $\partial W / \partial \xi=0$,

$$
\xi_{0} \simeq \beta q e E z_{\mathrm{top}}
$$

and

$$
W\left(\xi_{0}\right)=\frac{1}{\tilde{E}}\left[\operatorname{Ei}\left(\zeta_{0}\right)-\operatorname{Ei}\left(\zeta_{N}\right)+\log \left|\zeta_{0} / \zeta_{N}\right|\right],
$$

with $\operatorname{Ei}(x)$ being the exponential integral function, $\zeta_{0}=$ $\xi_{0}-\tilde{E}$, and $\zeta_{N}=\xi_{0}-\tilde{E} N$. (The complete expression for $\mathcal{Z}_{b}$ and the derivation details are given in the Appendix, Sec. 2). This yields the free energy $\bar{F}_{b}\left(z_{\text {top }}, N\right)=-k_{B} T \log \mathcal{Z}_{b}\left(z_{\text {top }}\right)$ associated with the bulk part of the chain without the account of counterions:

$$
\begin{aligned}
\beta \bar{F}_{b}\left(z_{\text {top }}, N\right) \approx & \beta U_{\text {sp }}\left(z_{\text {top }}\right)+\beta\left\langle H_{\text {self }, b}\right\rangle_{\psi} \\
& +\xi_{0} \tilde{z}_{\text {top }}-W\left(\xi_{0}\right)-N \log 2 \pi .
\end{aligned}
$$

The impact of counterions on the conformation of the bulk part of the chain may be estimated as a weak perturbation, so the bulk component of free energy reads

$$
F_{b}\left(z_{\text {top }}, N\right)=\bar{F}_{b}\left(z_{\text {top }}, N\right)+F_{\text {c ch }}\left(z_{\text {top }}, N\right),
$$

with

$$
F_{\mathrm{c} \mathrm{ch}}=\frac{4 \pi \sigma_{c} q e^{2} b}{\varepsilon \tilde{E}} \frac{e^{\tilde{E}\left(\tilde{z}_{\mathrm{top}}-\tilde{L}\right)}}{e^{\tilde{E} \tilde{z}_{\mathrm{top}} / N}-1}-\frac{\pi \sigma_{c} q e^{2} b}{\varepsilon} \tilde{z}_{\mathrm{top}} N .
$$

Here $L(\tilde{L}=L / b)$ is the size of the system in the $O Z$ direction, $S$ is its lateral area, and $e \sigma_{c}=e q N_{0} / S$ is the apparent surface charge density, associated with the counterions. The derivation of $F_{\mathrm{c} c h}$ is given in the Appendix, Sec. 3. As it may be seen from the above equation, the impact of the counterions on the chain conformation is small, provided $e \sigma_{c} / E \ll 1$ and $\tilde{E} \tilde{L} \gg 1$. Assuming that these conditions are fulfilled in the case of interest, Eq. (23) simplifies to

$$
\beta F_{\mathrm{cch}} \simeq-\frac{z_{\mathrm{top}}}{2 \mu} N
$$

where $\mu=1 / 2 \pi \sigma_{c} l_{B} q$ is the Gouy-Chapman length based on the apparent surface charge density $\sigma_{c}=q N_{0} / S$.

\section{B. Free energy of the adsorbed part of the chain}

Using the notation of the previous section, one can write the radius vector of $l$ th bead of the adsorbed part of the chain as $\mathbf{r}_{l}=\sum_{i=N_{0}}^{l} \mathbf{b}_{i}$. Then the radius vector that joins two ends 
of the adsorbed part reads

$$
\mathbf{R}=\sum_{i=N_{0}}^{N+1} \mathbf{b}_{i}=\sum_{s=1}^{N_{s}} \mathbf{d}_{s}
$$

where we introduce $\mathbf{d}_{s}=\mathbf{b}_{N_{0}+1-s}$ for the sake of notational simplicity. Obviously, for the adsorbed beads we have $\mathbf{r}_{k l}=$ $\sum_{s=k}^{l} \mathbf{d}_{s}$. Thus, the free energy of the adsorbed part may be written as

$$
\beta F_{s}=-\log \mathcal{Z}_{s}\left(N_{s}, \mathbf{R}\right),
$$

where $\mathcal{Z}_{s}\left(N_{s}, \mathbf{R}\right)$ is the conditional partition function

$$
\begin{aligned}
\mathcal{Z}_{s}\left(N_{s}, \mathbf{R}\right)= & \int_{0}^{2 \pi} d \phi_{1} \cdots \int_{0}^{2 \pi} d \phi_{N_{s}} e^{-\beta H_{\mathrm{self}, s}} \\
& \times \delta\left(\sum_{s=1}^{N_{s}} \mathbf{d}_{s}-\mathbf{R}\right) b^{2},
\end{aligned}
$$

where $\quad H_{\text {self }, s}=(1 / 2) \sum_{s_{1} \neq s_{2}} V\left(\mathbf{r}_{s_{1}}-\mathbf{r}_{s_{2}}\right)$ describes selfinteraction of the adsorbed monomers with the potential $V\left(\mathbf{r}_{i}-\mathbf{r}_{j}\right)$ defined in Eq. (10). The factor $b^{2}$ in the preceding equation keeps $\mathcal{Z}_{s}$ dimensionless. Since we assume that the adsorbed part of the chain forms a flat two-dimensional structure, the integration in Eq. (27) is performed over $N_{s}$ azimuthal angles $\phi_{1}, \ldots, \phi_{N_{s}}$, which define the directions of $N_{s}$ vectors $\mathbf{d}_{1}, \ldots, \mathbf{d}_{N_{s}}$ on the plane. Note that the evaluation of the conditional partition sum $\mathcal{Z}_{s}(\mathbf{R})$ also allows one to estimate the equilibrium configuration of the adsorbed part of the chain. Using as previously the integral representation of the $\delta$ function, we recast Eq. (27) in the form

$$
\begin{aligned}
\mathcal{Z}_{s}\left(N_{s}, \mathbf{R}\right) & \\
= & \int \frac{d \mathbf{p}}{(2 \pi)^{2}} b^{2} e^{-i \mathbf{p} \cdot \mathbf{R}} \int_{0}^{2 \pi} d \phi_{1} \cdots \int_{0}^{2 \pi} d \phi_{N_{s}} \\
& \times \exp \left\{-\frac{\beta}{2} \sum_{s_{1} \neq s_{2}} V\left(\mathbf{r}_{s_{1}}-\mathbf{r}_{s_{2}}\right)+i \mathbf{p} \cdot \sum_{s=1}^{N_{s}} \mathbf{d}_{s}\right\} \\
= & \int \frac{d \mathbf{p} b^{2}}{(2 \pi)^{2}} e^{-i \mathbf{p} \cdot \mathbf{R}_{\mathcal{Z}_{\mathrm{sp}}}(\mathbf{p})\left\langle\exp \left(-\frac{\beta}{2} \sum_{s_{1} \neq s_{2}} V\left(\mathbf{r}_{s_{1}}-\mathbf{r}_{s_{2}}\right)\right)\right\rangle_{\mathbf{p}},}
\end{aligned}
$$

where we define

$$
\begin{aligned}
\mathcal{Z}_{\mathrm{sp}}(\mathbf{p}) & =\int_{0}^{2 \pi} d \phi_{1} \cdots \int_{0}^{2 \pi} d \phi_{N_{s}} \exp \left(i \mathbf{p} \cdot \sum_{s=1}^{N_{s}} \mathbf{d}_{s}\right) \\
& =(2 \pi)^{N_{s}}\left[J_{0}(p b)\right]^{N_{s}} .
\end{aligned}
$$

Here $J_{0}(x)=(2 \pi)^{-1} \int_{0}^{2 \pi} \cos (x \cos \phi) d \phi$ is the zeroth-order Bessel function; we also take into account that $\mathbf{p} \cdot \mathbf{d}_{s}=$ $p b \cos \phi_{s}$. In Eq. (28) the average over the angles $\phi_{1}, \ldots, \phi_{N_{s}}$ is denoted by

$$
\begin{aligned}
\langle(\cdots)\rangle_{\mathbf{p}}= & \frac{1}{\mathcal{Z}_{\mathrm{sp}}(\mathbf{p})} \int_{0}^{2 \pi} d \phi_{1} \cdots \int_{0}^{2 \pi} d \phi_{N_{s}} \\
& \times \exp \left(i \mathbf{p} \cdot \sum_{s=1}^{N_{s}} \mathbf{d}_{s}\right)(\cdots) .
\end{aligned}
$$

Referring for computational details to the Appendix, Sec. 4, below we give the final result for the conditional partition function:

$$
\mathcal{Z}_{S}\left(N_{s}, \mathbf{R}\right)=\frac{(2 \pi)^{N_{s}}}{\pi N_{s}} e^{-R^{2} / N_{s} b^{2}-\pi \sqrt{2} q^{2} l_{B} N_{s}^{2} / R},
$$

where $R=|\mathbf{R}|$. From Eq. (26) then follows

$$
\begin{aligned}
\beta F_{s}\left(N_{s}, R\right)= & \frac{R^{2}}{N_{s} b^{2}}+\frac{\pi \sqrt{2} q^{2} l_{B} N_{s}^{2}}{R} \\
& -N_{s} \log 2 \pi-\log \pi N_{s} .
\end{aligned}
$$

Note that $N_{s}=N_{0}-N$. If we neglect the interaction of the adsorbed part of the chain with the bulk part we can estimate the equilibrium end-to-end distance of the adsorbed part $R$. Minimizing $F_{s}\left(N_{s}, R\right)$ with respect to $R$ and keeping $N_{s}$ fixed, $\left(\partial F_{s} / \partial R\right)_{N_{s}}=0$, we obtain the equilibrium value of $R$,

$$
R=\left(q^{2} b^{2} l_{B} \pi / \sqrt{2}\right)^{1 / 3} N_{s} .
$$

Equation (32) implies that the adsorbed part is stretched, $R \sim N_{s}$. Note that the condition of a stretched conformation does not necessarily imply a linearly stretched chain. Loose configurations of chaotic surface loops or circular conformations are also possible.

\section{Interaction between bulk and adsorbed parts of the chain}

The part of the free energy that accounts for interactions between the bulk part of the chain and the adsorbed part may be estimated as (see the Appendix, Sec. 5 for more detail)

$$
F_{b s}\left(N, z_{\text {top }}, R\right) \approx\left\langle H_{s b}\right\rangle_{N, z_{\text {top }}, R} .
$$

Here $H_{s b}$ is the interaction energy between $N$ charged monomers of the bulk part of the chain and $N_{s}=N_{0}-N$ monomers of the adsorbed part,

$$
\beta H_{s b}=\sum_{l=1}^{N} \sum_{m=1}^{N_{s}} \frac{l_{B}}{\left|\mathbf{r}_{l}-\mathbf{r}_{m}\right|}
$$

where $\mathbf{r}_{l}$ is the radius vector of the $l$ th monomer of the bulk part, $\mathbf{r}_{m}$ is that of the $m$ th monomers of the adsorbed part, and $\langle(\cdots)\rangle_{N, z_{\text {top }}, R}$ denotes the averaging at fixed $N, z_{\text {top }}$, and $R$. Using the definition of vectors $\mathbf{b}_{i}$ and $\mathbf{d}_{j}$, given in previous sections, we can write

$$
\mathbf{r}_{l}-\mathbf{r}_{m}=\sum_{s=l}^{N} \mathbf{b}_{s}+\sum_{s=1}^{m} \mathbf{d}_{s}
$$

and recast $H_{s b}$ in the form

$$
\begin{aligned}
\beta H_{s b}= & \sum_{l=1}^{N} \sum_{m=1}^{N_{s}} \int \frac{d \mathbf{k}}{(2 \pi)^{3}}\left(\frac{4 \pi l_{B}}{k^{2}}\right) \\
& \times \exp \left(i \mathbf{k} \cdot \sum_{s=l}^{N} \mathbf{b}_{s}+i \mathbf{k} \cdot \sum_{s=1}^{m} \mathbf{d}_{s}\right) .
\end{aligned}
$$

In Eq. (36) we again use the Fourier representation of the interaction potential $1 / r$ given in the Appendix, Sec. 1. Since the averaging is to be performed at fixed $N, z_{\text {top }}$, and $R$ we can 
approximate the exponential factor in (36) as

$$
\begin{gathered}
\left\langle\exp \left(i \mathbf{k} \cdot \sum_{s=l}^{N} \mathbf{b}_{s}+i \mathbf{k} \cdot \sum_{s=1}^{m} \mathbf{d}_{s}\right)\right\rangle_{N, z_{\text {top }}, R} \\
\approx \exp \left[-\frac{k_{\perp}^{2} b^{2}(N-l)}{4}\left(1-\frac{\tilde{z}_{\text {top }}^{2}}{N^{2}}\right)\right. \\
\left.+i k_{z} \frac{z_{\text {top }}}{N}(N-l)+i\left(\mathbf{k}_{\perp} \cdot \mathbf{R}\right) \frac{m}{N_{s}}\right]
\end{gathered}
$$

where we apply the same approximations as in Eqs. (13), (15), and (A3) for the bulk part of the chain and a similar one for the adsorbed part,

$$
\sum_{s=1}^{m} \mathbf{d}_{s} \approx \mathbf{R}\left(m / N_{s}\right)
$$

Substituting (37) into (36) and performing integration over $d \mathbf{k}$ (see the Appendix, Sec. 5 for details) we finally obtain

$$
\begin{aligned}
\beta F_{b s}= & \frac{l_{B} N N_{s}}{R}\left[\log \left(1+\sqrt{1+z_{\text {top }}^{* 2}}\right)\right. \\
& \left.+\frac{1}{z_{\text {top }}^{*}} \log \left(z_{\text {top }}^{*}+\sqrt{1+z_{\text {top }}^{* 2}}\right)-\log z_{\text {top }}^{*}\right] \\
& -\frac{l_{B} N}{R z_{\text {top }}^{*}} \log \left(2 N_{s} z_{\text {top }}^{*}\right),
\end{aligned}
$$

where $z_{\text {top }}^{*}=z_{\text {top }} / R$ characterizes the relative dimensions of the bulk and adsorbed parts of the chain.

\section{Dependence of the force and deformation on the external field}

Now we can determine the dependence on the electric field of the polyelectrolyte dimensions as well as the deformation of the target body. Simultaneously one obtains the dependence on applied field of the force that arises between the chain and target. This may be done by minimizing the total free energy of the system

$$
F\left(N, z_{\text {top }}, R\right)=F_{b}\left(N, z_{\text {top }}\right)+F_{s}\left(N_{s}, R\right)+F_{b s}\left(N, z_{\text {top }}, R\right)
$$

with respect to $N, z_{\text {top }}$, and $R$ and using $N_{s}=N_{0}-N$ and the constraint $z_{\text {top }} \leqslant b N$ (see the discussion above). The above three components of the free energy are given, respectively, by Eqs. (22), (31), and (39). This allows us to find $N, z_{\text {top }}$, and $R$ as functions of the applied electric field, that is, to obtain $N=N(E), z_{\text {top }}=z_{\text {top }}(E)$, and $R=R(E)$. Then one can compute the force acting on the target body. It reads

$$
\tilde{f}\left(\tilde{z}_{\text {top }}\right)=\tilde{E} \tilde{z}_{\text {top }}-\frac{\tilde{l}_{B} q^{2} N^{2}(\log N-1)}{\tilde{z}_{\text {top }}^{2}}-\frac{N}{2 \tilde{\mu}}+\frac{\partial \beta F_{b s}}{\partial \tilde{z}_{\text {top }}},
$$

where $\tilde{f}=\beta b f\left(z_{\text {top }}\right)$, with $f\left(z_{\text {top }}\right)=-\partial U_{\text {sp }} / \partial z_{\text {top }}$, is the reduced force for a particular force-deformation relation (8), and $\tilde{\mu}=\mu / b$ is the reduced Gouy-Chapman length. In Eq. (40) we exploit Eq. (19) for $\xi_{0}$ and the saddle point equation $i z_{\text {top }}-\partial W / \partial \xi=0$, valid for $\xi=\xi_{0}$ (see the Appendix, Sec. 2).

\section{MD SIMULATIONS}

We report MD simulations of a polyelectrolyte modeled by a freely jointed bead-spring chain of length $N_{0}+1$. The $\left(N_{0}+1\right)$ th end bead is uncharged and anchored to a planar surface at $z=0$. All the remaining $N_{0}$ beads carry one (negative) elementary charge. Electroneutrality of the system is fulfilled by the presence of $N_{0}$ monovalent free counterions of opposite charge, i.e., in our simulations $q=1$. For simplicity, we consider the counterions to have the same size as monomers. We also assume that the implicit solvent is a good one, which implies short-range purely repulsive interaction between all particles, described by a shifted Lennard-Jones potential. Neighboring beads along the chain are connected by a finitely extensible nonlinear elastic (FENE) potential. For the set of parameters used in our simulations, the bond length at zero force is $b \simeq \sigma_{\mathrm{LJ}}$, with $\sigma_{\mathrm{LJ}}$ the Lennard-Jones parameter. All particles except the anchor bead are exposed to a short-range repulsive interaction with the grafting plane at $z=0$ and with the upper boundary at $z=L_{z}$. The charged particles interact with the bare Coulomb potential. Its strength is quantified by the Bjerrum length $l_{B}=e^{2} / \varepsilon k_{B} T$. In the simulations we set $l_{B}=\sigma_{\mathrm{LJ}}$ and use a Langevin thermostat to hold the temperature $k_{B} T=\epsilon_{\mathrm{LJ}}$, with $\epsilon_{\mathrm{LJ}}$ the Lennard-Jones energy parameter. For more details of the simulation model and method see Refs. [23-25]. The free end of the chain is linked to a deformable target body, which is modeled by springs with various force-deformation relations. In this study we consider the two cases that seem to be the most important ones in terms of possible applications: linear and Hertzian springs described by Eqs. (1) and (3), respectively.

In the simulations we use two different setups: one where the spring is anchored at the top plane (Fig. 1, right panel) and another where the spring is attached to the grafting plane (Fig. 2, right panel). For simplicity, we assume that the anchor of springs is fixed and that they are aligned in the direction of the applied field, i.e., perpendicular to the grafting plane. Under these assumptions, the instantaneous length of the spring is $L-z_{\text {top }}$ in the first case and $z_{\text {top }}$ in the second one (see Figs. 1 and 2). Here we report simulation results obtained at total chain length $N_{0}=320$. The footprint of the simulation box is $L_{x} \times L_{y}=424 \times 424$ (in units of $\sigma_{\mathrm{LJ}}$ ) and the box height is $L_{z}=L=160$.

A typical simulation snapshot is shown in Fig. 3. We found that starting from relatively weak fields of $\mathrm{Eqeb} / k_{B} T \geqslant 0.1$ (recall that $q e$ is the monomer charge), the adsorbed part of the chain forms an almost flat two-dimensional structure. Small loops of the chain rise out of the plane up to a height of one monomer radius. The bulk part of the polyelectrolyte is strongly stretched in the perpendicular direction to the grafting plane with the interbead bonds being strongly aligned along the applied field. In sharp contrast to the field-free case [26-32], the counterion subsystem is practically decoupled from the polyelectrolyte, which drastically simplifies the analysis.

\section{RESULTS AND DISCUSSION}

In Figs. 4-8 we show results of MD simulations compared to the predictions of our theory. In particular, the spring length 


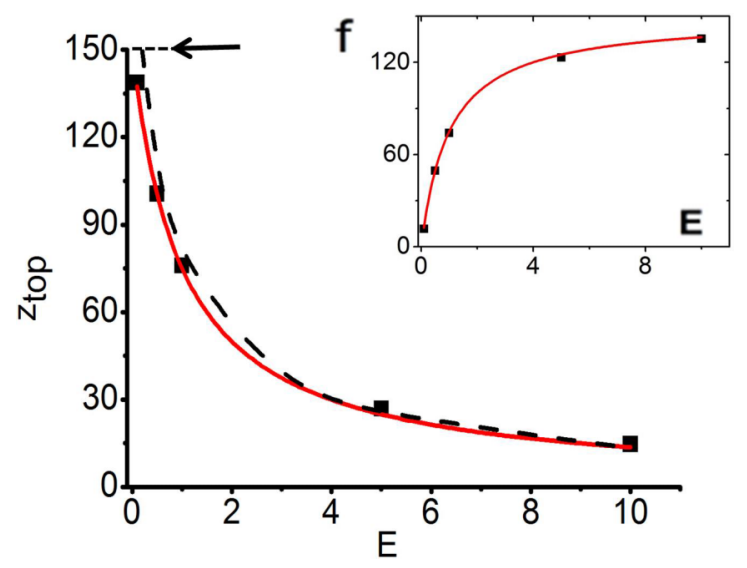

FIG. 4. End-point height $\tilde{z}_{\text {top }}=z_{\text {top }} / b$ of a chain linked to a linear spring, as a function of reduced applied field $\tilde{E}=q e E b / k_{B} T$. Lines show the results of the theory symbols the MD data. The dashed black line demonstrates $\tilde{z}_{\text {top }}=N$ of the previous simplified theory [13] with $N$ taken from the MD data. The inset shows the reduced force generated by the applied field $\tilde{f}=f b / k_{B} T$ as a function of reduced field. The bar equilibrium length of the spring is $h_{0}=10 \mathrm{~b}$ and its force constant is $\tilde{\kappa}=\kappa b^{2} / k_{B} T=1$. The length of the deformed spring reads $L_{z}-z_{\text {top }}=160 b-z_{\text {top }}$. The total length of the chain is $N_{0}=320$. The arrow indicates $z_{\text {top }}$ for the undeformed spring.

and magnitude of the induced force are shown as functions of the applied electric field. The spring length characterizes the deformation of the target body caused by the force acting from the polyelectrolyte chain. Figure 4 refers to a linear spring anchored to the upper wall. Figures 5-9 show the behavior of Hertzian springs of different bare equilibrium lengths (i.e., of colloidal particles of different size); these springs are anchored to the lower wall. The figures clearly demonstrate the very good agreement between theory and MD data obtained in our study. We wish to stress the lack of any fitting parameters used in these

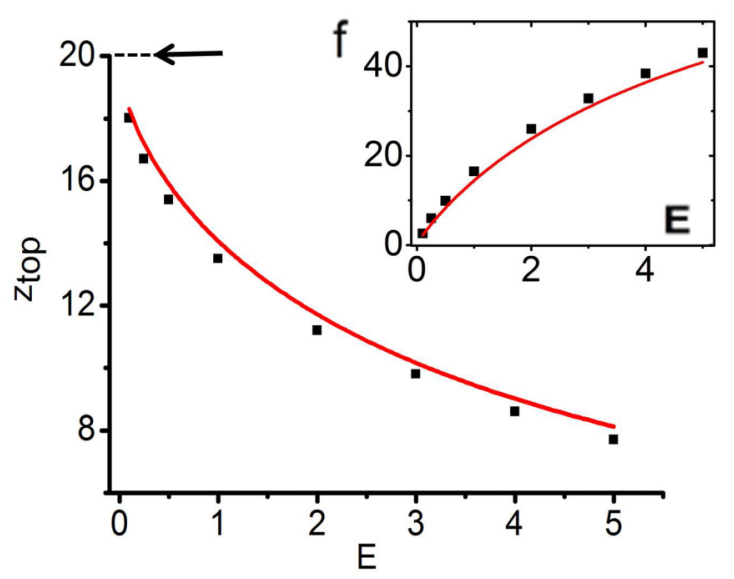

FIG. 5. Reduced length of a Hertzian spring $\tilde{z}=z_{\text {top }} / b$ as a function of reduced field $\tilde{E}=q e E b / k_{B} T$. Lines show the results of the theory and symbols the MD data. The inset shows the reduced force generated by the applied field $\tilde{f}=f b / k_{B} T$ as a function of field. The bare equilibrium length of the Hertzian spring (undeformed colloidal particle) is $z_{\text {top }, 0}=d_{c}=20 b$ and the force constant is $\tilde{\kappa}=\kappa b^{5 / 2} / k_{B} T=1$. The total length of the chain is $N_{0}=320$. The arrow indicates $z_{\text {top }}$ for the undeformed spring.

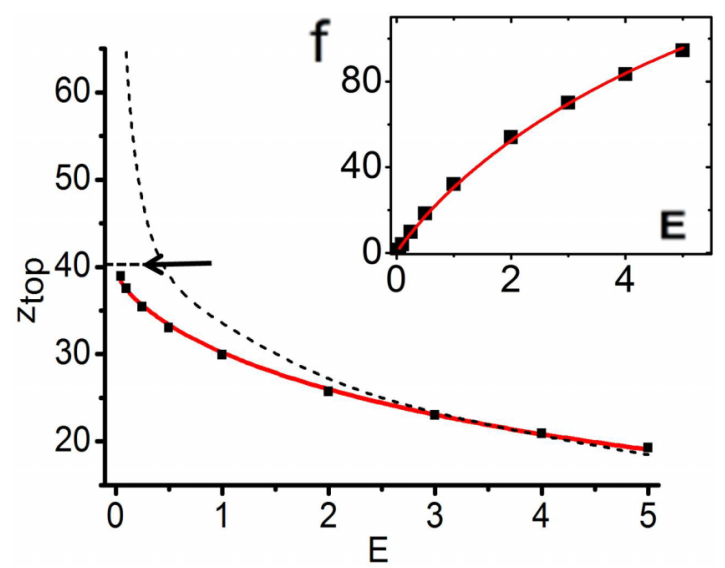

FIG. 6. Same as Fig. 5, but for $z_{\text {top }, 0}=d_{c}=40 b$. The dashed black line demonstrates $\tilde{z}_{\text {top }}=N$ of the previous simplified theory [13] with $N$ taken from the MD data.

plots. Note, however, that the theory has been developed for a highly charged chain with a relatively strong self-interaction and interaction with the charged plane. This results in an almost flat two-dimensional structure of the adsorbed part of the chain and small transversal fluctuations of the bulk part; the bond vectors of the bulk part cannot be directed down. Although the theory is rather accurate, some systematic deviations are observed for very small fields and for the shortest Hertzian springs with $z_{\text {top }, 0}=20 \mathrm{~b}$. In the latter case the deformation of the spring and the force acting on a target body are slightly underestimated. This possibly happens since the condition $N \gg 1$ is not as accurate for short springs as for long ones.

The theory also underestimates the number of monomer beads $N$ in a bulk for small fields. While the theory is rather accurate when $\tilde{E}>1$, there occur noticeable deviations from MD data at small fields $\tilde{E}<1$ (see Fig. 9). Fortunately, this deficiency of the present theory with respect to $N$ does not degrade the accuracy of the theoretical dependences $z_{\text {top }}(E)$ and $f(E)$, which seem to be the most important quantities in terms of possible applications. It is noteworthy that for aqueous solutions at the ambient conditions, the characteristic units of force and field are $k_{B} T / b \approx k_{B} T / l_{B} \approx 6 \mathrm{pN}$ and $k_{B} T / b e \approx k_{B} T / l_{B} e \approx 35 \mathrm{~V} / \mu \mathrm{m}$, respectively. The latter

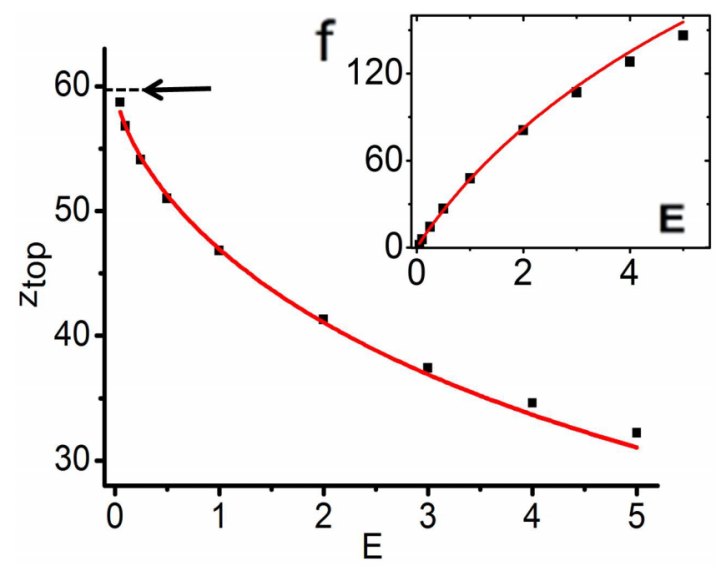

FIG. 7. Same as Fig. 5, but for $z_{\text {top }, 0}=d_{c}=60 b$. 


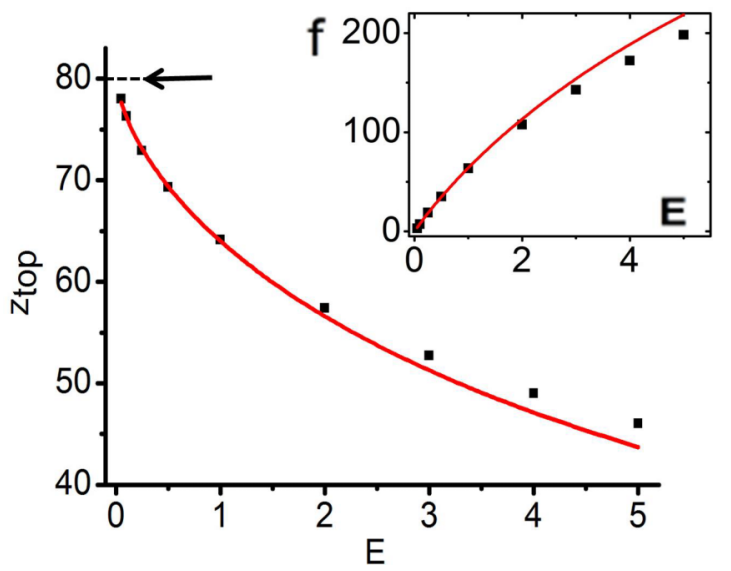

FIG. 8. Same as Fig. 5, but for $z_{\text {top }, 0}=d_{c}=80 b$.

value is about one order of magnitude smaller than the critical breakdown field for water [33]. Another feature is worth noting. While the electric field is altered within a relatively narrow range, the magnitude of the resulting force varies over a rather wide range, which is clearly of great interest for applications.

It is also noteworthy to compare the theoretical results of the present study with the corresponding results of the previous simplified theory (see Ref. [13]). Some representative examples are shown in Figs. 4 and 6. Obviously the simplified theory is accurate for linear springs, except at small fields $\tilde{E}<1$. At the same time it fails to satisfactorily describe the behavior of Hertzian springs. The simplified theory drastically underestimates deformation of a target body at small fields $(\tilde{E}<0.5)$, noticeably underestimates it at intermediate range $(1<\tilde{E}<3)$, and overestimates it in strong fields $(\tilde{E}>4)$. The simplified theory has an acceptable accuracy only in a rather narrow field interval.

The phenomenon addressed in the present study may be used in future nanomachinery: A prototype of a possible nanodevice, which may be called a nanovice or nanonippers, is illustrated in Fig. 2. Here the contraction of two polyelectrolyte

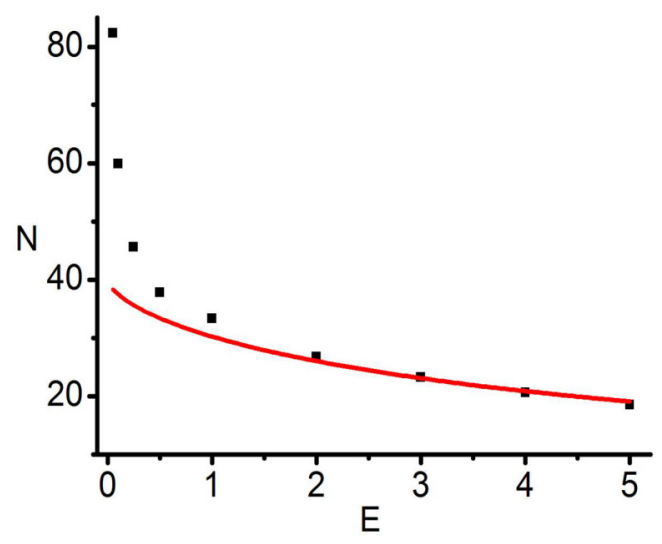

FIG. 9. Number of chain monomers in the bulk $N$ as a function of reduced applied field $\tilde{E}=q e E b / k_{B} T$. Lines show the results of the theory and symbols the MD data. The length of the undeformed Hertzian spring is $z_{\text {top }, 0}=40 b$ and the force constant is $\tilde{\kappa}=\kappa b^{5 / 2} / k_{B} T=1$. The total length of the chain is $N_{0}=320$. chains in an external electric field allows one to fix firmly a colloidal particle, which would otherwise perform Brownian motion. At zero or weak fields the particle will be released. Using our theory, one can compute the magnitude of the field needed to keep the particle fixed, although additional knowledge about the intensity of the Brownian motion and friction forces is required. Naturally, one can think about other nanosize objects, e.g., viruses, cellular organelles, or small bacteria. These objects would be characterized by other force-deformation relations.

Consider, for example, nanovices in aqueous solutions at the ambient conditions with $l_{B}=0.7 \mathrm{~nm}$. For simplicity we analyze the case of only one chain (see the right panel of Fig. 2); the generalization for a few chains is straightforward. Let the polyelectrolyte chain be flexible and consist of $N_{0}=180$ monomers of size $b \approx l_{B}$, each carrying a charge $1 e$. Let the colloidal particle be of diameter $d_{0}=50 \mathrm{~b}=35 \mathrm{~nm}$. If we use the Young modulus $Y=0.01 \mathrm{GP}$, as for rubber [34], for the particle material and $v=0.1$ for the Poisson ratio, we obtain $\tilde{\kappa}=2.86 .^{2}$ In this case the field $\tilde{E}=1$ of about $35 \mathrm{~V} / \mu \mathrm{m}$ generates a force of about $240 \mathrm{pN}$ and the relative deformation of $\Delta d / d_{0}=0.123$. Forty-four monomers remain in the bulk and 136 are adsorbed. If the field increases up to $\tilde{E}=2$, that is, up to $70 \mathrm{~V} / \mu \mathrm{m}$, the force increases to about $440 \mathrm{pN}$, with the deformation of $\Delta d / d_{0}=0.186$ and 41 monomers in the bulk.

Naturally, there exist plenty of other possible applications of the mechanism studied, which we plan to address in future research.

\section{CONCLUSION}

We analyzed the generation of a mechanical force by an external electric field, applied to a grafted polyelectrolyte that is linked to a deformable target body. We developed a theory of this phenomenon and performed MD simulations. The case of strong electrostatic self-interaction of the chain and its interaction with the charged plane was addressed. We considered target bodies with two different force-deformation relations, which seem to be the most important for possible applications: (i) a linear relation and (ii) that of a Hertzian spring. The first relation models the behavior of a coiled Gaussian chain, while the second one represents that of a squeezed colloidal particle. The theoretical dependences of the generated force and of the compression of the target body are in very good agreement with the simulation data. The theory, however, underestimates the number of beads $N$ of the bulk part of the chain for weak fields and small sizes of colloidal particles. Interestingly, the generated force strongly depends on the applied electric field. While the magnitude of the force varies over a wide interval, the field itself alters within a rather narrow range only. The phenomenon addressed here may play an important role in future nanomachinery. For instance, it could be utilized to design vicelike devices (nanovices and nanonippers) that keep nanosized objects fixed. Other

\footnotetext{
${ }^{2}$ The Hertzian force $F_{H}$ depends on the Young modulus $Y$, Poisson ratio $\nu$, radius of particle $R$, and deformation $\xi$ as $F_{H}=\kappa \xi^{3 / 2}=$ $\frac{2}{3} \frac{Y \sqrt{R}}{\left(1-v^{2}\right)} \xi^{3 / 2}$ (see, e.g., $[19,20]$ ).
} 
applications of this phenomenon, which require manipulations with nano-objects, such as fusing them together by an applied pressure, are also possible.

\section{ACKNOWLEDGMENT}

This work was supported by a grant from the President of the RF (Grant No. MK-2823.2015.3).

\section{APPENDIX}

Here we present some calculational detail of quantities derived in the main text.

\section{Computation of $\left\langle\boldsymbol{H}_{\mathrm{self}, b}\right\rangle_{\psi}$}

First we show that $H_{\text {self, } b}$ given in Eq. (10) may written in the form (11). Using the integral representation of the $\delta$ function

$$
\delta(\mathbf{r})=(2 \pi)^{-d} \int e^{i \mathbf{k} \cdot \mathbf{r}} d \mathbf{k},
$$

where $d$ is the dimension of the vector $\mathbf{r}$, we write

$$
\begin{aligned}
\frac{1}{\left|\mathbf{r}_{l m}\right|} & =\frac{1}{(2 \pi)^{3}} \int d \mathbf{x} \int d \mathbf{k} e^{i \mathbf{k} \cdot\left(\mathbf{r}_{l m}-\mathbf{x}\right)} \frac{1}{|\mathbf{x}|}=\frac{1}{(2 \pi)^{3}} \int d \mathbf{k}\left(\frac{4 \pi}{k^{2}}\right) \exp \left(i \mathbf{k} \cdot \sum_{s=l}^{m} \mathbf{b}_{s}\right) \\
& =\int \frac{d \mathbf{k}}{(2 \pi)^{3}}\left(\frac{4 \pi}{k^{2}}\right) \exp \left(i \sum_{s=l}^{m} \mathbf{k}_{\perp} \cdot \mathbf{b}_{s}^{\perp}+k_{z} b_{s}^{z}\right),
\end{aligned}
$$

where $4 \pi / k^{2}$ is the Fourier transform of $1 /|\mathbf{x}|$. Summation of $\left|\mathbf{r}_{l m}\right|^{-1}$ with the prefactor $q^{2} e^{2} / 2 \varepsilon$ over all $l, m=1, \ldots, N$ yields Eq. (11).

To find $\left\langle H_{\text {self }, b}\right\rangle_{\psi}$ in Eq. (14) first we compute the average

$$
\left\langle\exp \left(i \sum_{s=s_{1}}^{s_{2}} \mathbf{k}_{\perp} \cdot \mathbf{b}_{s}^{\perp}\right)\right\rangle_{\psi}=\frac{1}{(2 \pi)^{N}} \int_{0}^{2 \pi} d \psi_{1} \cdots \int_{0}^{2 \pi} d \psi_{N} \exp \left(i \sum_{s=s_{1}}^{s_{2}} \mathbf{k}_{\perp} \cdot \mathbf{b}_{s}^{\perp}\right) .
$$

Due to the lateral symmetry, we choose the direction of the vector $\mathbf{k}_{\perp}$ along the $O X$ axis to obtain

$$
\begin{aligned}
\left\langle\exp \left(i \mathbf{k}_{\perp} \cdot \sum_{s=s_{1}}^{s_{2}} \mathbf{b}_{s}^{\perp}\right)\right\rangle_{\psi} & =\frac{1}{(2 \pi)^{N}} \int_{0}^{2 \pi} d \psi_{1} \cdots \int_{0}^{2 \pi} d \psi_{N} \exp \left(i k_{\perp} b \sum_{s=s_{1}}^{s_{2}} \cos \psi_{s} \sin \theta_{s}\right) \\
& =\prod_{s=s_{1}}^{s_{2}} \int_{0}^{2 \pi} \frac{d \psi_{s}}{2 \pi} e^{i k_{\perp} b \cos \psi_{s} \sin \theta_{s}}=\prod_{s=s_{1}}^{s_{2}} J_{0}\left(k_{\perp} b \sin \theta_{s}\right)=\exp \left[\sum_{s=s_{1}}^{s_{2}} \log J_{0}\left(k_{\perp} b \sin \theta_{s}\right)\right] \\
& \simeq \exp \left[\sum_{s=s_{1}}^{s_{2}} \log \left(1-k_{\perp}^{2} b^{2} \sin ^{2} \theta_{s} / 4\right)\right] \approx \exp \left[-\sum_{s=s_{1}}^{s_{2}} \frac{k_{\perp}^{2} b^{2} \sin ^{2} \theta_{s}}{4}\right] \\
& \approx \exp \left[-\frac{k_{\perp}^{2} b^{2}\left|s_{1}-s_{2}\right|}{4}\left(1-\frac{\tilde{z}_{\text {top }}^{2}}{N^{2}}\right)\right]
\end{aligned}
$$

where we use the approximation $\cos ^{2} \theta_{s} \approx \tilde{z}_{\text {top }}^{2} / N^{2}$ and keep in the Bessel function expansion only the leading terms $J_{0}(x)=$ $1-x^{2} / 4+\cdots$, where $x \sim k$. The latter approximation is justified since the main contribution from the integrand in (14) is accumulated in the vicinity of $k=0$.

Using now the approximation

$$
\sum_{s=s_{1}}^{s_{2}} \eta_{s} \approx \frac{\tilde{z}_{\text {top }}\left(s_{2}-s_{1}\right)}{N}
$$

and substituting it together with (A3) into Eq. (14), we obtain

$$
\beta\left\langle H_{\text {self }, b}\right\rangle_{\psi}=\frac{\beta q^{2} e^{2}}{2 \varepsilon} \sum_{s_{1} \neq s_{2}} \int \frac{d \mathbf{k}}{(2 \pi)^{3}}\left(\frac{4 \pi}{k_{\perp}^{2}+k_{z}^{2}}\right) \exp \left[-\frac{k_{\perp}^{2} b^{2}\left|s_{1}-s_{2}\right|}{4}\left(1-\frac{\tilde{z}_{\text {top }}^{2}}{N^{2}}\right)\right] e^{i k_{z} \tilde{z}_{\text {top }}\left|s_{1}-s_{2}\right| / N} .
$$

In this expression, one can integrate over $\mathbf{k}$ (first, over $k_{z}$, using residues) to get the result

$$
\beta\left\langle H_{\text {self }, b}\right\rangle_{\psi}=\frac{l_{B} q^{2}}{2} \sum_{s_{1} \neq s_{2}} \frac{\sqrt{\pi}}{2 h} e^{g^{2} / 4 h^{2}} \operatorname{Erfc}\left(\frac{g}{2 h}\right),
$$


where $h^{2}=b^{2}\left|s_{1}-s_{2}\right|\left(1-\tilde{z}_{\text {top }}^{2} / N^{2}\right) / 4$ and $g=\left|s_{1}-s_{2}\right| \tilde{z}_{\text {top }} / N$. Since $\tilde{z}_{\text {top }} \sim N$ and $\left|s_{1}-s_{2}\right| \sim N \gg 1$, it is easy to show that $g / 2 h \gg 1$. With $e^{x^{2}} \operatorname{Erfc}(x)=(\sqrt{\pi} x)^{-1}$ for $x \gg 1$ we obtain

$$
\beta\left\langle H_{\mathrm{self}, b}\right\rangle_{\psi}=\frac{l_{B} q^{2} N}{2 z_{\mathrm{top}}} \sum_{s_{1} \neq s_{2}} \frac{1}{\left|s_{1}-s_{2}\right|} \simeq \frac{l_{B} q^{2} N}{z_{\mathrm{top}}} \int_{1}^{N-1} d s_{1} \int_{s_{1}+1}^{N} \frac{d s_{2}}{s_{2}-s_{1}} \simeq \frac{l_{B} q^{2} N^{2}}{z_{\mathrm{top}}}(\log N-1),
$$

that is, Eq. (16) of the main text.

\section{Computation of $\mathcal{Z}_{b}\left(z_{\text {top }}\right)$}

From Eqs. (17) and (13) it follows that $W(\xi)$ is defined as

$$
W(\xi)=\log \int_{0}^{1} d \eta_{1} \cdots \int_{0}^{1} d \eta_{N} \exp \left\{\sum_{s=1}^{N}(i \xi-\tilde{E} s) \eta_{s}\right\}=\log \prod_{s=1}^{N} \int_{0}^{1} d \eta_{s} e^{(i \xi-\tilde{E} s) \eta_{s}} \simeq \int_{1}^{N} d s\left[\log \left(e^{i \xi-\tilde{E} s}-1\right)-\log (i \xi-\tilde{E} s)\right]
$$

The integral $\int_{-\infty}^{+\infty} d \xi \exp \left[-i \xi \tilde{z}_{\text {top }}+W(\xi)\right]$ in Eq. (17) may be estimated with the use of the steepest-descent method, that is, using the fact that for large $N$ the value of $\tilde{z}_{\text {top }}$ is also large, $z_{\text {top }} / b \gg 1$. Then the saddle point equation reads

$$
\frac{d}{d \xi}\left[-i \xi \tilde{z}_{\mathrm{top}}+W(\xi)\right]=-i \tilde{z}_{\mathrm{top}}+i \int_{1}^{N} d s\left[\frac{e^{i \xi-\tilde{E} s}}{e^{i \xi-\tilde{E} s}-1}-\frac{1}{i \xi-\tilde{E} s}\right]=0
$$

With the new variable $\xi_{0}=i \xi$, we obtain the equation that defines the implicit dependence of $\xi_{0}$ on $\tilde{z}_{\text {top }}$ and $N$ :

$$
\tilde{z}_{\text {top }}=\frac{1}{\tilde{E}}\left[\log \frac{e^{\xi_{0}-\tilde{E}}-1}{\xi_{0}-\tilde{E}}-\log \frac{e^{\xi_{0}-\tilde{E} N}-1}{\xi_{0}-\tilde{E} N}\right] .
$$

For $N \gg 1$, one can find rather accurately the solution of Eq. (A10). Indeed, the assumption that $\xi_{0} \sim 1 \ll N$ leads to the conclusion that $z_{\text {top }} \sim \log N$, which may not hold true for either the coiled chain or for the chain stretched by the force. On the other hand the assumption $\xi_{0} \sim N$, which yields $\xi_{0}-\tilde{E} \sim N$, implies that one can apply the approximation log $\left[\left(e^{x}-1\right) / x\right] \simeq$ $x-\log x$ at $x \gg 1$. Using the evident condition $\xi_{0}-\tilde{E} \gg \xi_{0}-\tilde{E} N$ one obtains

$$
\tilde{E} \tilde{z}_{\text {top }} \simeq \xi_{0}-\tilde{E}-\log \left(\xi_{0}-\tilde{E}\right)
$$

or

$$
\xi_{0} \simeq\left(\tilde{z}_{\text {top }}+1\right) \tilde{E}+\log \tilde{z}_{\text {top }} \tilde{E}
$$

If we again take into account that $\tilde{z}_{\text {top }} \sim N \gg 1$ and $\tilde{E} \sim 1 \ll N$ we arrive at an even more simple solution for $\xi_{0}$,

$$
\xi_{0} \simeq \tilde{E} \tilde{z}_{\text {top }}
$$

Hence we obtain the approximate expression of the partition sum

$$
\mathcal{Z}_{b}\left(z_{\text {top }}\right) \approx(2 \pi)^{N-1} e^{-\beta U_{s}-\beta\left\langle H_{\text {self }, b}\right\rangle_{\psi}} \exp \left(-\xi_{0} \tilde{z}_{\text {top }}+W\left(\xi_{0}\right)-\frac{1}{2} \log \frac{\left|W^{\prime \prime}\left(\xi_{0}\right)\right|}{2 \pi}\right),
$$

with $\xi_{0}$ given in the preceding equation and with $W\left(\xi_{0}\right)$ defined by Eq. (A8). It may be written as

$$
W\left(\xi_{0}\right)=(1 / \tilde{E})\left[\operatorname{Ei}\left(\zeta_{0}\right)+\log \left|\zeta_{0} / \zeta_{N}\right|-\operatorname{Ei}\left(\zeta_{N}\right)\right]
$$

where $\operatorname{Ei}(x)$ is the exponential integral function and we abbreviate $\zeta_{0}=\xi_{0}-\tilde{E}$ and $\zeta_{N}=\xi_{0}-\tilde{E} N$. Similarly, we write $W^{\prime \prime}$ as

$$
W^{\prime \prime}\left(\xi_{0}\right)=\tilde{E}^{-1}\left[\frac{e^{\zeta_{N}}}{e^{\zeta_{N}}-1}-\frac{e^{\zeta_{0}}}{e^{\zeta_{0}}-1}-\frac{1}{\zeta_{N}}+\frac{1}{\zeta_{0}}\right] .
$$

Finally, we obtain the free energy $\bar{F}_{b}\left(z_{\text {top }}, N\right)$ associated with the bulk part of the chain (without taking into account counterions)

$$
\beta \bar{F}_{b}\left(z_{\text {top }}, N\right) \approx \beta U_{\text {sp }}\left(z_{\text {top }}\right)+\beta\left\langle H_{\text {self }, b}\right\rangle_{\psi}-N \log 2 \pi+\xi_{0} z_{\text {top }}-W\left(\xi_{0}\right)+\log \left|W^{\prime \prime}\left(\xi_{0}\right)\right|^{1 / 2} .
$$

Note that for $N \gg 1$ the term containing $W^{\prime \prime}\left(\xi_{0}\right)$ is logarithmically small as compared to other terms and may be neglected. 


\section{Free energy of counterions}

The results of the MD simulations show that the counterions are well separated from the chain if the field and volume of the systems are not very small. Therefore, the impact of the counterions on the chain conformation may be treated as a small perturbation. Here we perform simple estimates of the free energy of counterions. We can approximate it as

$$
F_{\text {count }} \simeq F_{c c}+F_{c E}+F_{\mathrm{cch}},
$$

where $F_{c c}$ is the free energy associated with the counterion-counterion interactions, $F_{c E}$ refers to the free energy of the counterions interactions with the external field $E$, and $F_{\mathrm{cch}}$ is the free energy associated with the charged chain. In the case of interest one can neglect the dependence of $F_{c c}$ and $F_{c E}$ on the chain conformation, so we do not need to compute these terms. At the same time $F_{\mathrm{c} c h}$ can be estimated as the electrostatic energy of the chain in the additional potential $\varphi_{c}(z)$ caused by counterions,

$$
F_{\mathrm{cch}} \approx \sum_{i=1}^{N}-q e \varphi_{c}\left(z_{i}\right)
$$

To find $\varphi_{c}(z)$ we start with the equilibrium Boltzmann distribution of counterions $\rho_{c}(z)$ in the external field $E$ neglecting their self-interaction:

$$
\rho_{c}(z)=\rho_{0} e^{q e E z / k_{B} T}=\frac{N_{0} e q E}{S k_{B} T} e^{q e E(z-L) / k_{B} T},
$$

where $L$ is the size of the system in the direction along $O Z$ and $S$ is its lateral area. To obtain constant $\rho_{0}$ in the preceding equation, we apply the normalization condition $S \int_{0}^{L} \rho_{c}(z) d z=N_{0}$. Next we compute the electric field $E_{c}$ due to counterions, performing the same derivation as for the electric field of a uniformly charged plane

$$
\begin{aligned}
E_{c}(z) & =\frac{q e}{\varepsilon} \int_{0}^{L} d z_{1} \rho_{c}\left(z_{1}\right) \int_{0}^{2 \pi} d \phi \int_{0}^{\infty} \frac{\partial}{\partial z} \frac{r d r}{\sqrt{\left(z_{1}-z\right)^{2}+r^{2}}}=\frac{2 \pi e q N_{0}}{\varepsilon S} e^{-q e E L / k_{B} T}\left[2 e^{q e E z / k_{B} T}-e^{q e E L / k_{B} T}-1\right] \\
& =\left(4 \pi e \sigma_{c} / \varepsilon\right) e^{\tilde{E}(\tilde{z}-\tilde{L})}-\left(2 \pi e \sigma_{c} / \varepsilon\right),
\end{aligned}
$$

where $\sigma_{c}=q N_{0} / S$ corresponds to the apparent surface charge density due to counterions and $\tilde{L}=L / b$. The second term in Eq. (A16), $2 \pi e \sigma_{c} / \varepsilon$, corresponds to the renormalization of the external field $E$ due to the counterion screening of the upper plane $E \rightarrow E-2 \pi e \sigma_{c} / \varepsilon$. From Eq. (A16), finally we get the additional potential

$$
\varphi_{c}(z)=2 \pi e \sigma_{c} z / \varepsilon-\left(4 \pi e \sigma_{c} b / \varepsilon \tilde{E}\right) e^{\tilde{E}(\tilde{z}-\tilde{L})} .
$$

Substituting Eq. (A17) into Eq. (A15) we obtain

$$
F_{\mathrm{cch}}=-\sum_{i=1}^{N} \frac{2 \pi \sigma_{c}}{\varepsilon} q e^{2} z_{i}+\frac{4 \pi e \sigma_{c} b}{\varepsilon \tilde{E}} e^{-\tilde{E} \tilde{L}} \sum_{i=1}^{N} e^{-\tilde{E} \tilde{z}_{i}} .
$$

Using $\tilde{z}_{i}=\sum_{s=i}^{N} \cos \theta_{s}$ [see Eq. (7)] along with the approximation $\cos \theta_{s}=\overline{\cos \theta_{s}}=\tilde{z}_{\text {top }} / N$, we find for the first and second terms in Eq. (A17)

$$
\begin{gathered}
\frac{4 \pi e \sigma_{c} b}{\varepsilon \tilde{E}} e^{-\tilde{E} \tilde{L}} \sum_{i=1}^{N} e^{-\tilde{E} \tilde{z}_{i}}=\frac{4 \pi e \sigma_{c} b}{\varepsilon \tilde{E}} \frac{e^{\tilde{E}\left(\tilde{z}_{\mathrm{top}}-\tilde{L}\right)}}{e^{\tilde{E} \tilde{z}_{\mathrm{top}} / N}-1}, \\
\sum_{i=1}^{N} \sum_{s=1}^{N} \frac{2 \pi \sigma_{c} q e^{2} b}{\varepsilon} \cos \theta_{s}=\frac{\pi \sigma_{c} q e^{2} b}{\varepsilon} \tilde{z}_{\mathrm{top}} N
\end{gathered}
$$

which yields Eq. (23) of the main text.

\section{Computation of $\mathcal{Z}_{s}(\mathbf{R})$} write

We start with the computation of $\left\langle e^{-\beta H_{\text {self,s }}}\right\rangle_{\mathbf{p}}$. Using only the first-order term in the cumulant expansion of the exponent we

$$
\left\langle\exp \left(-\frac{\beta}{2} \sum_{s_{1} \neq s_{2}} V\left(\mathbf{r}_{s_{1}}-\mathbf{r}_{s_{2}}\right)\right)\right\rangle_{\mathbf{p}} \approx \exp \left(-\frac{\beta}{2} \sum_{s_{1} \neq s_{2}}\left\langle V\left(\mathbf{r}_{s_{1}}-\mathbf{r}_{s_{2}}\right)\right\rangle_{\mathbf{p}}\right) .
$$

This is a mean-field approximation, which is usually a good approximation for systems with long-range interactions. Since $V(r)$ refers to the unscreened Coulomb interactions, we expect this approximation to be rather accurate.

Similar to Eq. (A1) we can write

$$
V\left(\mathbf{r}_{s_{1}}-\mathbf{r}_{s_{2}}\right)=\int \frac{d \mathbf{k}}{(2 \pi)^{3}} \tilde{V}(k) e^{i \mathbf{k} \cdot\left(\mathbf{r}_{s_{1}}-\mathbf{r}_{s_{2}}\right)},
$$


where $\tilde{V}(k)=\left(q^{2} e^{2} / \varepsilon\right)\left(4 \pi / k^{2}\right)$ is the Fourier transform of the interaction potential. This yields

$$
\begin{aligned}
\left\langle V\left(\mathbf{r}_{s_{1}}-\mathbf{r}_{s_{2}}\right)\right\rangle_{\mathbf{p}} & =\frac{1}{\mathcal{Z}_{0}(\mathbf{p})} \int_{0}^{2 \pi} d \phi_{1} \cdots \int_{0}^{2 \pi} d \phi_{N_{s}} \exp \left(i \mathbf{p} \cdot \sum_{s=1}^{N_{s}} \mathbf{d}_{s}\right) V\left(\mathbf{r}_{s_{1}}-\mathbf{r}_{s_{2}}\right)=\int \frac{d \mathbf{k}}{(2 \pi)^{3}} \tilde{V}(k)\left\langle e^{i \mathbf{k} \cdot\left(\mathbf{r}_{s_{1}}-\mathbf{r}_{s_{2}}\right)}\right\rangle_{\mathbf{p}} \\
& =\frac{1}{\mathcal{Z}_{0}(\mathbf{p})} \int_{0}^{2 \pi} d \phi_{1} \cdots \int_{0}^{2 \pi} d \phi_{N_{s}} \int \frac{d \mathbf{k}}{(2 \pi)^{3}} \tilde{V}(k) \exp \left(i \mathbf{p} \cdot \sum_{s=1}^{N_{s}} \mathbf{d}_{s}+i \mathbf{k}_{\perp} \cdot \sum_{s_{1}}^{s_{2}} \mathbf{d}_{l}\right) \\
& =\int \frac{d \mathbf{k}}{(2 \pi)^{3}} \tilde{V}(k)\left[\frac{J_{0}\left(\left|\mathbf{k}_{\perp}+\mathbf{p}\right| b\right)}{J_{0}(p b)}\right]^{\left|s_{2}-s_{1}\right|} .
\end{aligned}
$$

Here we take into account that $\mathbf{p}$ is a two-dimensional vector and use the definition (29) of $\mathcal{Z}_{0}(\mathbf{p})$. Substituting Eq. (A21) into Eq. (28) we arrive at

$$
\begin{aligned}
\mathcal{Z}_{s}(\mathbf{R}) & \approx(2 \pi)^{N_{s}} \int \frac{d \mathbf{p}}{(2 \pi)^{2}} e^{-i \mathbf{p} \cdot \mathbf{R}}\left[J_{0}(p b)\right]^{N_{s}} \exp \left(-\frac{\beta}{2} \int \frac{d \mathbf{k}}{(2 \pi)^{3}} \tilde{V}(k) \sum_{s_{1} \neq s_{2}}\left[\frac{J_{0}\left(\left|\mathbf{k}_{\perp}+\mathbf{p}\right| b\right)}{J_{0}(p b)}\right]^{\left|s_{1}-s_{2}\right|}\right) \\
& =(2 \pi)^{N_{s}} \int \frac{d \mathbf{p}}{(2 \pi)^{2}} \exp \left(-i \mathbf{p} \cdot \mathbf{R}+N_{s} \log \left[J_{0}(p b)\right]-\frac{\beta}{2} \int \frac{d \mathbf{k}}{(2 \pi)^{3}} \tilde{V}(k) \sum_{s_{1} \neq s_{2}}\left[\frac{J_{0}\left(\left|\mathbf{k}_{\perp}+\mathbf{p}\right| b\right)}{J_{0}(p b)}\right]^{\left|s_{1}-s_{2}\right|}\right) \\
& \simeq(2 \pi)^{N_{s}} \int \frac{d \mathbf{p}}{(2 \pi)^{2}} \exp \left(-i \mathbf{p} \cdot \mathbf{R}-\frac{1}{4} N_{s} p^{2} b^{2}-\frac{\beta}{2} \int \frac{d \mathbf{k}}{(2 \pi)^{3}} \tilde{V}(k) \sum_{s_{1} \neq s_{2}}\left[\frac{J_{0}\left(\left|\mathbf{k}_{\perp}+\mathbf{p}\right| b\right)}{J_{0}(p b)}\right]^{\left|s_{1}-s_{2}\right|}\right) .
\end{aligned}
$$

Using the new integration variable

$$
\mathbf{G}=\mathbf{p}-\frac{2 i \mathbf{R}}{N_{s} b^{2}},
$$

we obtain

$$
\begin{aligned}
\mathcal{Z}_{s}(\mathbf{R}) & =(2 \pi)^{N_{s}} e^{-R^{2} / N_{s} b^{2}} \int \frac{d \mathbf{G}}{(2 \pi)^{2}} e^{-N_{s} b^{2} G^{2} / 4} \exp \left\{-\frac{\beta}{2} \sum_{s_{1} \neq s_{2}} \int \frac{d \mathbf{k}}{(2 \pi)^{3}} \tilde{V}(k) \exp \left[\left|s_{2}-s_{1}\right| \log \left(\frac{J_{0}\left(\left|\mathbf{k} \perp+\mathbf{G}+\frac{2 i \mathbf{R}}{N_{s} b^{2}}\right| b\right)}{J_{0}\left(\left|\mathbf{G}+\frac{2 i \mathbf{R}}{N_{s} b^{2}}\right| b\right)}\right)\right]\right\} \\
& \simeq(2 \pi)^{N_{s}} e^{-R^{2} / N_{s} b^{2}} \int \frac{d \mathbf{G}}{(2 \pi)^{2}} e^{-N_{s} b^{2} G^{2} / 4} \exp \left\{-\frac{\beta}{2} \sum_{s_{1} \neq s_{2}} \int \frac{d \mathbf{k}}{(2 \pi)^{3}} \tilde{V}(k) \exp \left[\left|s_{2}-s_{1}\right| \log \left(\frac{J_{0}\left(\left|\mathbf{k}_{\perp}+\frac{2 i \mathbf{R}}{N_{s} b^{2}}\right| b\right)}{\left.\left.J_{0}\left(\frac{2 i|\mathbf{R}|}{N_{s} b}\right)\right]\right\}}\right)\right]\right\} \\
& \approx(2 \pi)^{N_{s}} \frac{1}{\pi N_{s} b^{2}} e^{-R^{2} / N_{s} b^{2}-\beta W_{1}(R)} .
\end{aligned}
$$

To derive Eq. (A23) we take into account that since $N_{s} \gg 1$, only values of $G \sim 1 / b \sqrt{N_{s}}$ contribute to the above integral. The analysis also shows that $R \sim N_{s} b$ [see Eq. (32)], which allows us to neglect $\mathbf{G}$ as compared to (R/ $\left.N_{s}\right) b^{2}$ and to perform the Gaussian integration in the last line of (A23). Furthermore, we define

$$
\begin{aligned}
\beta W_{1}(R) & \simeq \frac{\beta}{2} \sum_{s_{1} \neq s_{2}} \int \frac{d \mathbf{k}}{(2 \pi)^{3}} \tilde{V}(k) e^{-i \mathbf{k}_{\perp} \cdot \mathbf{R}\left|s_{2}-s_{1}\right| / N_{s}} e^{-\mathbf{k}_{\perp}^{2} b^{2}\left|s_{1}-s_{2}\right| / 4} \\
& \approx \frac{q^{2} l_{B}}{2 \pi^{2}} \int_{1}^{N_{s}-1} d s_{1} \int_{s_{1}+1}^{N_{s}} d s_{2} \int_{-\infty}^{\infty} d k_{z} \int d \mathbf{k}_{\perp} \frac{e^{-i \mathbf{k}_{\perp} \cdot \mathbf{R}\left|s_{2}-s_{1}\right| / N_{s}} e^{-\mathbf{k}_{\perp}^{2} b^{2}\left|s_{1}-s_{2}\right| / 4}}{k_{z}^{2}+\mathbf{k}_{\perp}^{2}},
\end{aligned}
$$

where we use again the expansion of $J_{0}(x)$ and keep only the leading term. Integration over $k_{z}$ may be easily performed, yielding $\pi / k_{\perp}$. Hence we obtain

$$
\begin{gathered}
\int_{-\infty}^{\infty} d k_{z} \int d \mathbf{k}_{\perp} \frac{e^{-i \mathbf{k}_{\perp} \cdot \mathbf{R}\left|s_{2}-s_{1}\right| / N_{s}} e^{-\mathbf{k}_{\perp}^{2} b^{2}\left|s_{1}-s_{2}\right| / 4}}{k_{z}^{2}+\mathbf{k}_{\perp}^{2}}=\pi \int_{0}^{\infty} d k_{\perp} e^{-b^{2}\left|s_{2}-s_{1}\right| k_{\perp}^{2} / 4} \int_{0}^{2 \pi} e^{-i \cos \phi k_{\perp} R\left|s_{2}-s_{1}\right| / N_{s}} d \phi \\
=2 \pi^{2} \int_{0}^{\infty} e^{-b^{2}\left|s_{2}-s_{1}\right| k_{\perp}^{2} / 4} J_{0}\left(\frac{k_{\perp} R\left|s_{2}-s_{1}\right|}{N_{s}}\right) d k_{\perp}=\pi^{5 / 2} \frac{e^{-R^{2}\left|s_{2}-s_{1}\right| / 2 N_{s}^{2} b^{2}}}{b \sqrt{\left|s_{2}-s_{2}\right|}} I_{0}\left(\frac{R^{2}\left|s_{2}-s_{1}\right|}{2 N_{s}^{2} b^{2}}\right),
\end{gathered}
$$

where $I_{0}(x)$ is the modified Bessel function of the first kind. Substituting the above result into Eq. (A24), we observe that since $R \sim b N_{s}$, the main contribution in the integrals over $s_{1}$ and $s_{2}$ comes from the region where $\left|s_{2}-s_{1}\right|$ is small; here we can 
approximate $I_{0}(x) \approx 1 .{ }^{3}$ Therefore, we can write

$$
\beta W_{1}(R) \approx q^{2} \tilde{l}_{B} \sqrt{\pi} N_{s}^{3 / 2} \int_{0}^{1} d x \int_{x}^{1} d y \frac{e^{-\left(R^{2} / 2 N_{s} b^{2}\right)|y-x|}}{\sqrt{|y-x|}}=q^{2} \tilde{l}_{B} \sqrt{\pi} N_{s}^{3 / 2} H\left(\frac{R^{2}}{2 N_{s} b^{2}}\right) .
$$

Here the function $H(x)$ reads

$$
H(x)=\frac{\sqrt{\pi} \operatorname{erf}(\sqrt{x})(x-1 / 2)+x e^{-x}}{x^{3 / 2}} ;
$$

it behaves as $H(x) \simeq \sqrt{\frac{\pi}{x}}$ for $x \gg 1$. Hence, for $R^{2} \gg N_{s} b^{2}$ we obtain

$$
\beta W_{1}(R)=\frac{\pi \sqrt{2} q^{2} l_{B} N_{s}^{2}}{R}
$$

and finally, the conditional partition function

$$
\mathcal{Z}_{S}(\mathbf{R}) \simeq \frac{(2 \pi)^{N_{s}}}{\pi N_{s}} e^{-R^{2} / N_{s} b^{2}-\pi \sqrt{2} q^{2} l_{B} N_{s}^{2} / R}
$$

\section{Calculation of $F_{b s}\left(N, z_{\text {top }}, R\right)$}

The conditional free energy of the system $F\left(N, z_{\text {top }}, R\right)$ may be written in the following form:

$$
\begin{aligned}
e^{-\beta F\left(N, z_{\mathrm{top}}, R\right)}= & \int_{0}^{2 \pi} d \psi_{1} \cdots d \psi_{N} \int_{0}^{1} d \cos \theta_{1} \cdots \int_{0}^{1} d \cos \theta_{N} \delta\left(z_{\mathrm{top}}-b \sum_{s=1}^{N} \cos \theta_{s}\right) b \\
& \times \int_{0}^{2 \pi} d \phi_{1} \cdots d \phi_{N_{s}} \delta\left(\sum_{s=1}^{N_{s}} \mathbf{d}_{s}-\mathbf{R}\right) b^{2} e^{-\beta U_{\mathrm{sp}}\left(z_{\mathrm{top}}\right)-\beta H_{\mathrm{ext}}-\beta H_{\mathrm{sel}, b}-\beta H_{\mathrm{self}, s}-\beta H_{b s}} \\
= & \int d \Gamma_{b} e^{-\beta H_{1}} \int d \Gamma_{s} e^{-\beta H_{2}} \frac{\int d \Gamma_{b} \int d \Gamma_{s} e^{-\beta\left(H_{1}+H_{2}\right)} e^{-\beta H_{b s}}}{\int d \Gamma_{b} \int d \Gamma_{s} e^{-\beta\left(H_{1}+H_{2}\right)}} \\
= & e^{-\beta F_{b}\left(N, z_{\mathrm{top}}\right)} e^{-\beta F_{s}\left(N_{s}, R\right)}\left\langle e^{-\beta H_{\mathrm{bs}}}\right\rangle_{N, z_{\mathrm{top}}, R} \approx e^{-\beta F_{b}\left(N, z_{\mathrm{top}}\right)} e^{-\beta F_{s}\left(N_{s}, R\right)} e^{-\beta\left\langle H_{b s}\right\rangle_{N, z_{\mathrm{top}}, R},}
\end{aligned}
$$

which yields Eq. (5) of the main text:

$$
F\left(N, z_{\text {top }}, R\right) \approx F_{b}\left(N, z_{\text {top }}\right)+F_{s}\left(N_{s}, R\right)+F_{b s}\left(N, z_{\text {top }}, R\right) .
$$

Here $F_{b s}\left(N, z_{\text {top }}, R\right)=\left\langle H_{b s}\right\rangle_{N, z_{\text {top }}, R}$. In Eq. (A29) we introduce the shorthand notation

$$
\int d \Gamma_{b}=\int_{0}^{2 \pi} d \psi_{1} \cdots \int_{0}^{2 \pi} d \psi_{N} \int_{0}^{1} d \cos \theta_{1} \cdots \int_{0}^{1} d \cos \theta_{N}, \quad \int d \Gamma_{s}=\int_{0}^{2 \pi} d \phi_{1} \cdots d \phi_{N_{s}}
$$

as well as

$$
e^{-\beta H_{1}}=e^{-\beta U_{\mathrm{sp}}\left(z_{\mathrm{top}}\right)-\beta H_{\mathrm{ext}}-\beta H_{\mathrm{self}, b}} \delta\left(z_{\mathrm{top}}-b \sum_{s=1}^{N} \cos \theta_{s}\right) b ; \quad e^{-\beta H_{2}}=e^{-\beta H_{\mathrm{self}, s}} \delta\left(\sum_{s=1}^{N_{s}} \mathbf{d}_{s}-\mathbf{R}\right) b^{2} .
$$

To compute $\left\langle H_{b s}\right\rangle_{N, z_{\text {top }}, R}$ we use as previously the approximation of small transverse fluctuations for the bulk part of the chain $H_{b s} \approx\left\langle H_{b s}\right\rangle_{\psi}$. With this approximation one can write

$$
\left\langle\exp \left(i \mathbf{k} \cdot \sum_{s=l}^{N} \mathbf{b}_{s}+i \mathbf{k} \cdot \sum_{s=1}^{m} \mathbf{d}_{s}\right)\right\rangle_{N, z_{\mathrm{top}}, R} \approx\left\langle\exp \left(i \mathbf{k}_{\perp} \cdot \sum_{s=l}^{N} \mathbf{b}_{s}^{\perp}\right)\right\rangle_{\psi}\left\langle\exp \left(i k_{z} b \cdot \sum_{s=l}^{N} \eta_{s}+i \mathbf{k}_{\perp} \cdot \sum_{s=1}^{m} \mathbf{d}_{s}\right)\right\rangle_{N, z_{\mathrm{top}}, R},
$$

with the same notation as above. The first factor on the right-hand side of Eq. (A30) may be computed as in Eq. (A3), yielding

$$
\left\langle\exp \left(i \mathbf{k}_{\perp} \cdot \sum_{s=l}^{N} \mathbf{b}_{s}^{\perp}\right)\right\rangle_{\psi}=\exp \left[-\frac{k_{\perp}^{2} b^{2}(N-l)}{4}\left(1-\frac{\tilde{z}_{\text {top }}^{2}}{N^{2}}\right)\right]=e^{-k_{\perp}^{2} h_{1}^{2}} .
$$

\footnotetext{
${ }^{3}$ More precisely, the function $e^{-x^{2}} I_{0}(x)$ is rather close to $e^{-x^{2}}$ when $x=R^{2}\left|s_{2}-s_{1}\right| / N_{s}^{2} b^{2}$ is of the order of unity; this guarantees that the discussed approximation has an acceptable accuracy.
} 
Using the same approximation as in Eqs. (A4) and (38),

$$
b \sum_{s=l}^{N} \eta_{s} \approx \frac{z_{\text {top }}}{N}(N-l)=g_{1}, \quad \sum_{s=1}^{m} \mathbf{d}_{s}=\frac{m}{N_{s}} \mathbf{R}=\mathbf{R}^{\prime},
$$

we arrive at Eq. (37), which we write as

$$
\left\langle\exp \left(i \mathbf{k} \cdot \sum_{s=l}^{N} \mathbf{b}_{s}+i \mathbf{k} \cdot \sum_{s=1}^{m} \mathbf{d}_{s}\right)\right\rangle_{N, z_{\text {top }}, R}=e^{-k_{\perp}^{2} h_{1}^{2}+i k_{z} g_{1}+i \mathbf{k}_{\perp} \cdot \mathbf{R}^{\prime}},
$$

where $h_{1}, g_{1}$, and $\mathbf{R}^{\prime}$ have been defined in the above equations.

Below we give the calculational detail of Eq. (39) where we need to compute the integral in Eq. (36) with the substitute from (37). With the above notation for $h_{1}, g_{1}$, and $\mathbf{R}^{\prime}$ it may be written as

$$
\frac{1}{(2 \pi)^{3}} \int \frac{4 \pi}{k_{\perp}^{2}+k_{z}^{2}} e^{-k_{\perp}^{2} h_{1}^{2}+i k_{z} g_{1}+i \mathbf{k}_{\perp} \cdot \mathbf{R}^{\prime}} d \mathbf{k} .
$$

First we compute the integral over $k_{z}$ using the residue at $k_{z}=i k_{\perp}$ :

$$
\int_{-\infty}^{\infty} \frac{4 \pi}{k_{\perp}^{2}+k_{z}^{2}} e^{i k_{z} g_{1}} d k_{z}=\frac{4 \pi^{2}}{k_{\perp}} e^{-k_{\perp} g_{1}} .
$$

Next the integration over $\mathbf{k}_{\perp}$ may be performed to yield

$$
\begin{aligned}
& \frac{4 \pi^{2}}{8 \pi^{3}} \int_{0}^{\infty} k_{\perp} d k_{\perp} \frac{e^{-k_{\perp}^{2} h_{1}^{2}-k_{\perp} g_{1}}}{k_{\perp}} \int_{0}^{2 \pi} e^{i k_{\perp} R^{\prime} \cos \phi} d \phi \\
& \quad=\int_{0}^{\infty} e^{-k_{\perp}^{2} h_{1}^{2}-k_{\perp} g_{1}} J_{0}\left(k_{\perp} R^{\prime}\right) d k_{\perp}=\frac{1}{R^{\prime}} \int_{0}^{\infty} e^{-z^{2}\left(h_{1} / R^{\prime}\right)^{2}-z\left(g_{1} / R^{\prime}\right)} J_{0}(z) d z \simeq \frac{1}{\sqrt{R^{\prime 2}+g_{1}^{2}}},
\end{aligned}
$$

where we take into account that $g_{1} / R^{\prime} \gg h_{1} / R^{\prime}$ for $N \sim N_{s} \gg 1$.

Using the above result for the integral over $\mathbf{k}$ we can find $\left\langle H_{b s}\right\rangle_{N, z_{\text {top }}, R}$,

$$
\beta\left\langle H_{b s}\right\rangle_{N, z_{\text {top }}, R} \simeq l_{B} \int_{1}^{N} d l \int_{1}^{N_{s}} \frac{d m}{\sqrt{\frac{z_{\text {top }}^{2}(N-l)^{2}}{N^{2}}+R^{2} \frac{m^{2}}{N_{s}^{2}}}}=\frac{l_{B} N N_{s}}{z_{\text {top }}} \log Z_{1}+\frac{l_{B} N N_{s}}{R} \log Z_{2}+\frac{l_{B} N}{z_{\text {top }}} \log Z_{3},
$$

where

$$
\begin{gathered}
Z_{1}=\left(z_{\mathrm{top}} / R\right)+\sqrt{1+\left(z_{\mathrm{top}} / R\right)^{2}}, \\
Z_{2}=\left(R / z_{\mathrm{top}}\right)\left[1+\sqrt{1+\left(z_{\mathrm{top}} / R\right)^{2}}\right], \\
Z_{3}=\frac{R}{2 z_{\mathrm{top}} N_{s}}
\end{gathered}
$$

and we use definitions of $g_{1}$ and $R^{\prime}$ and approximate the summation over $l$ and $m$ by the integration. After simple algebra we arrive at the expression (39) for $\left\langle H_{b s}\right\rangle_{N, z_{\text {top }}, R}$.

[1] M. Muthukumar, J. Chem. Phys. 86, 7239 (1987).

[2] A. K. Bajpai, Prog. Polym. Sci. 22, 523 (1997).

[3] O. V. Borisov, E. B. Zhulina, and T. M. Birshtein, J. Phys. (France) II 4, 913 (1994).

[4] I. Borukhov, D. Andelman, and H. Orland, Macromolecules 31, 1665 (1998).

[5] X. Chatellier and J.-F. Joanny, Phys. Rev. E 57, 6923 (1998).

[6] M. Muthukumar, J. Chem. Phys. 120, 9343 (2004).

[7] A. V. Dobrynin, A. Deshkovski, and M. Rubinstein, Phys. Rev. Lett. 84, 3101 (2000).

[8] A. V. Dobrynin, A. Deshkovski, and M. Rubinstein, Macromolecules 34, 3421 (2001).
[9] O. V. Borisov, F. A. M. Leermakers, G. J. Fleer, and E. B. Zhulina, J. Chem. Phys. 114, 7700 (2001).

[10] R. R. Netz, Phys. Rev. Lett. 90, 128104 (2003).

[11] C. Friedsam, H. E. Gaub, and R. R. Netz, Europhys. Lett. 72, 844 (2005).

[12] N. V. Brilliantov and C. Seidel, Europhys. Lett. 97, 28006 (2012).

[13] C. Seidel, Y. A. Budkov, and N. Brilliantov, Nanoeng. Nanosyst. 227, 142 (2013).

[14] R. R. Netz, J. Phys. Chem. B 107, 8208 (2003).

[15] O. V. Borisov, A. B. Boulakh, and E. B. Zhulina, Eur. Phys. J. E 12, 543 (2003). 
[16] P. Podgornik and B. Jonsson, Europhys. Lett. 24, 501 (1993).

[17] P. Podgornik, T. Akesson, and B. Jonsson, J. Chem. Phys. 102, 9423 (1995).

[18] P. Podgornik and M. Licer, Curr. Opin. Colloid Interface Sci. 11, 273 (2006).

[19] H. Kuninaka and H. Hayakawa, Phys. Rev. E 79, 031309 (2009).

[20] K. Saitoh, A. Bodrova, H. Hayakawa, and N. V. Brilliantov, Phys. Rev. Lett. 105, 238001 (2010).

[21] A. Y. Grosberg and A. R. Khokhlov, Statistical Physics of Macromolecules (AIP, Woodbury, 1994).

[22] Y. A. Budkov, C. Seidel, and N. Brilliantov (unpublished).

[23] N. A. Kumar and C. Seidel, Phys. Rev. E 76, 020801(R) (2007).

[24] F. S. Csajka and C. Seidel, Macromolecules 33, 2728 (2000).

[25] N. A. Kumar and C. Seidel, Macromolecules 38, 9341 (2005).

[26] R. G. Winkler, M. Gold, and P. Reineker, Phys. Rev. Lett. 80, 3731 (1998).
[27] N. V. Brilliantov, D. V. Kuznetsov, and R. Klein, Phys. Rev. Lett. 81, 1433 (1998).

[28] R. Golestanian, M. Kardar, and T. B. Liverpool, Phys. Rev. Lett. 82, 4456 (1999).

[29] H. Schiessel and P. Pincus, Macromolecules 31, 7953 (1998).

[30] U. Micka, C. Holm, and K. Kremer, Langmuir 15, 4033 (1999).

[31] A. Diehl, M. C. Barbosa, and Y. Levin, Phys. Rev. E 54, 6516 (1996).

[32] A. Naji and R. R. Netz, Phys. Rev. Lett. 95, 185703 (2005).

[33] M. Zahn, Y. Ohki, D. B. Fenneman, R. J. Gripshover, and V. H. Gehman, Jr., Proc. IEEE 74, 1182 (1986).

[34] The Engineering ToolBox, Modulus of Elasticity or Young's Modulus and Tensile Modulus for some Common Materials, 2012, available at http://www.engineeringtoolbox.com/youngmodulus-d_417.html 